\title{
NONLOCAL POROUS MEDIUM EQUATION: BARENBLATT PROFILES AND OTHER WEAK SOLUTIONS
}

\author{
PIOTR BILER, CYRIL IMBERT, AND GRZEGORZ KARCH
}

\begin{abstract}
A degenerate nonlinear nonlocal evolution equation is considered; it can be understood as a porous medium equation whose pressure law is nonlinear and nonlocal. We show the existence of sign changing weak solutions to the corresponding Cauchy problem. Moreover, we construct explicit compactly supported selfsimilar solutions which generalize Barenblatt profiles - the well-known solutions of the classical porous medium equation.
\end{abstract}

\section{INTRODUCTION}

In this work, we study the following degenerate nonlinear nonlocal evolution equation

$$
\partial_{t} u=\nabla \cdot\left(|u| \nabla^{\alpha-1}\left(|u|^{m-2} u\right)\right), \quad x \in \mathbb{R}^{d}, t>0,
$$

where $m>1$ and $\nabla^{\alpha-1}$ denotes the integro-differential operator $\nabla(-\Delta)^{\frac{\alpha}{2}-1}, \alpha \in(0,2)$. The equation is supplemented with an initial condition

$$
u(0, x)=u_{0}(x) .
$$

First, we construct nonnegative self-similar solutions of equation (1.1) which are explicit and compactly supported. They generalize the classical Barenblatt-Kompaneets-Pattle-Zel'dovich solutions of the porous medium equation, see (1.4) below. Second, we prove the existence of sign changing weak solutions to problem (1.1) -(1.2) for merely integrable initial data, and we prove that these solutions satisfy sharp hypercontractivity $L^{1} \mapsto L^{p}$ estimates.

A nonlocal operator. Equation (1.1) involves a nonlocal operator denoted by $\nabla^{\alpha-1}$ which can be defined as the Fourier multiplier whose symbol is $i \xi|\xi|^{\alpha-2}$. This notation emphasizes that it is a (pseudo-differential) operator of order $\alpha-1$. Recalling the definition of the fractional Laplace operator $(-\Delta)^{\frac{\alpha}{2}}(v)=\mathcal{F}^{-1}\left(|\xi|^{\alpha} \mathcal{F} v\right)$ and the Riesz potential $\mathcal{I}_{\beta}=(-\Delta)^{-\frac{\beta}{2}}$, i.e. Fourier multipliers whose symbols are $|\xi|^{\alpha}$ and $|\xi|^{-\beta}$ respectively (see for instance [23, Ch. V]), the fractional gradient $\nabla^{\alpha-1}$ can also be written as $\nabla \mathcal{I}_{2-\alpha}$. Finally, let us emphasize that the definition of $\nabla^{\alpha-1}$ is consistent with the usual gradient: $\nabla^{1}=\nabla$; the components of $\nabla^{0}$ are the Riesz transforms; moreover we have $\nabla \cdot \nabla^{\alpha-1}=\nabla^{\frac{\alpha}{2}} \cdot \nabla^{\frac{\alpha}{2}}=-(-\Delta)^{\frac{\alpha}{2}}$. It is also possible, following the reasoning from

Date: August 8, 2018.

2000 Mathematics Subject Classification. 35K55, 35B45, 35C06.

Key words and phrases. porous medium equation, nonlocal equation, hypercontractivity, self-similar solutions.

The authors wish to thank Jean Dolbeault and Régis Monneau for the fruitful discussions they had together. The authors were supported by an EGIDE project (PHC POLONIUM 20078TL, 0185, 2009-2010). The second author was supported by an ANR project (EVOL). The first and the third authors were supported by the MNSzW grant N201 418839, and the Foundation for Polish Science operated within the Innovative Economy Operational Programme 2007-2013 funded by European Regional Development Fund (Ph.D. Programme: Mathematical Methods in Natural Sciences). 
[12, Th. 1] to define the fractional gradient via the singular integral formula for smooth and bounded functions $v: \mathbb{R}^{d} \rightarrow \mathbb{R}$

$$
\nabla^{\alpha-1} v(x)=C_{d, \alpha} \int(v(x)-v(x+z)) \frac{z}{|z|^{d+\alpha}} \mathrm{d} z
$$

with a suitable constant $C_{d, \alpha}>0$

Related equations and results. Our preliminary results for the problem (1.1)-(1.2) have been announced in 3 .

First, we would like to shed light on the link between (1.1) and other partial differential equations. Notice that when $\alpha=2$ equation (1.1) coincides with the classical (nonlinear parabolic) porous medium equation

$$
\partial_{t} u=\nabla \cdot\left(|u| \nabla\left(|u|^{m-2} u\right)\right)=\nabla \cdot\left((m-1)|u|^{m-1} \nabla u\right)
$$

For the theory of porous media equations, the interested reader is referred to [27, 28] and references therein. Of course, for $m=2$, the Boussinesq equation is recovered.

The following nonlinear and nonlocal equation

$$
\partial_{t} v+\left|v_{x}\right|\left(-\frac{\partial^{2}}{\partial x^{2}}\right)^{\frac{\alpha}{2}} v=0
$$

in the one-dimensional case $x \in \mathbb{R}$ was studied by the first, the third authors and R. Monneau [4]. Such an equation was derived as a model for the dynamics of dislocations in crystals. In [4, the existence, uniqueness and comparison properties of (viscosity) solutions have been proved, and explicit self-similar solutions have been constructed. Notice that the function $u=v_{x}$, where $v$ is a solution to (1.5), solves the one-dimensional case of (1.1) with $m=2$. Thus, equation (1.1) is a multidimensional generalization of the one in (1.5).

Recently, Caffarelli and Vázquez [5, 7] studied nonnegative weak solutions of (1.1) in the case $m=2$ in the multidimensional case. Precisely, they studied the following (nonlocal) porous medium equation in $\mathbb{R}^{d}$

$$
\partial_{t} u=\nabla \cdot(u \nabla p)
$$

with the nonlocal pressure law $p=(-\Delta)^{-s} u, 0<s<1$, obtained from the density $u \geq 0$. Notice that, for $\alpha=2-2 s \in(0,2)$, equation (1.6) reads $\partial_{t} u=\nabla \cdot\left(u \nabla^{\alpha-1} u\right)$. For sign changing $u$ 's, our equation (1.1) is a (formally parabolic) extension of equation (1.6) of the structure of (1.5). In [5], Caffarelli and Vázquez constructed nonnegative weak solutions for (1.6), i.e. for (1.1) with $m=2$, with initial data satisfying: $u_{0} \in$ $L^{1}\left(\mathbb{R}^{d}\right) \cap L^{\infty}\left(\mathbb{R}^{d}\right)$ and such that $0 \leq u_{0}(x) \leq A \mathrm{e}^{-a|x|}$ for some $A, a>0$. Besides the positivity and the mass preservation, the properties of solutions, listed in the next paper [7, p. 4], include the finite speed of propagation proved using the comparison with suitable supersolutions. Further regularity properties of solutions of (1.1) with $m=2$ and $\alpha \in(0,1)$ are studied in [6].

Another nonlocal porous medium equation has been proposed in 10, 11, 29

$$
\partial_{t} u+(-\Delta)^{\frac{\alpha}{2}}\left(|u|^{m-1} u\right)=0
$$


for $\alpha=1$ and $\alpha \in(0,2)$, respectively. Among several other properties like smoothing effects and decay estimates, solutions of this generalization of the porous medium equation enjoy the $L^{1}$-contraction property, so, they are unique. But self-similar solutions are not compactly supported [29, Th. 1.1].

Finally, we recall that the following nonlocal higher order equation, appearing in the modeling of propagation of fractures in rocks,

$$
\partial_{t} u=\nabla \cdot\left(u^{n} \nabla(-\Delta)^{\frac{1}{2}} u\right)
$$

(with $u \geq 0$ and $n>1$ ), has been studied in [15] in a one-dimensional bounded domain. At least formally, this equation with $n=1$ corresponds to (1.1) with $\alpha=3$ and $m=2$.

Notation. In this work, $Q_{T}$ denotes $(0, T) \times \mathbb{R}^{d}$. The usual norm of the Lebesgue space $L^{p}\left(\mathbb{R}^{d}\right)$ is denoted by $\|\cdot\|_{p}$ for any $p \in[1, \infty]$, and $H^{s, p}\left(\mathbb{R}^{d}\right)$ with the norm $\|\cdot\|_{H^{s, p}}$ is the fractional order Sobolev space, see Section 3. The Fourier transform $\mathcal{F}$ and its inverse transform $\mathcal{F}^{-1}$ of a function $v \in L^{1}\left(\mathbb{R}^{d}\right)$ are defined by

$$
\mathcal{F} v(\xi)=(2 \pi)^{-\frac{d}{2}} \int v(x) \mathrm{e}^{-i x \cdot \xi} \mathrm{d} x, \quad \mathcal{F}^{-1} v(x)=(2 \pi)^{-\frac{d}{2}} \int v(x) \mathrm{e}^{i x \cdot \xi} \mathrm{d} \xi .
$$

Here, all integrals with no integration limits are over the whole space $\mathbb{R}^{d}$ if one integrates with respect to $x$ and over the whole half-line $\mathbb{R}^{+}=[0, \infty)$ if the integration is with respect to $t$. As usual, $w_{+}=\max \{0, w\}$, $w_{-}=\max \{0,-w\}$, so $w=w_{+}-w_{-}$. Constants (always independent of $x$ and $t$ ) will be denoted by the same letter $C$, even if they may vary from line to line. Sometimes we write, e.g., $C=C(p, q, r)$ when we want to emphasize the dependence of $C$ on particular parameters $p, q, r$, for instance.

\section{MAIN RESULTS}

In this work, we show two main results: we construct explicit self-similar solutions of equation (1.1), as well as we prove that the initial value problem (1.1)-(1.2) has a global-in-time weak solution which satisfies certain optimal decay estimates.

We first "recall" the appropriate notion of weak solutions for Equation (1.1), see for instance [27, 28.

Definition 2.1 (Weak solutions). A function $u: Q_{T} \rightarrow \mathbb{R}$ is a weak solution of the problem (1.1)-(1.2) in $Q_{T}$ if $u \in L^{1}\left(Q_{T}\right), \nabla^{\alpha-1}\left(|u|^{m-2} u\right) \in L_{\mathrm{loc}}^{1}\left(Q_{T}\right)$ and $|u| \nabla^{\alpha-1}\left(|u|^{m-2} u\right) \in L_{\mathrm{loc}}^{1}\left(Q_{T}\right)$, and

$$
\iint\left(u \partial_{t} \varphi-|u| \nabla^{\alpha-1}\left(|u|^{m-2} u\right) \cdot \nabla \varphi\right) \mathrm{d} t \mathrm{~d} x+\int u_{0}(x) \varphi(0, x) \mathrm{d} x=0
$$

for all test functions $\varphi \in \mathcal{C}^{\infty}\left(Q_{T}\right) \cap \mathcal{C}\left(\overline{Q_{T}}\right)$ such that $\varphi$ has a compact support in the space variable $x$ and vanishes near $t=T$.

The first main result of this work says that there is a family of nonnegative explicit compactly supported self-similar solutions of (1.1), i.e. nonnegative solutions that are invariant under a suitable scaling. Observe that if $u(t, x)$ is a solution of (1.1), then so is $L^{d \lambda} u\left(L t, L^{\lambda} x\right)$ for each $L>0$, where $\lambda=(d(m-1)+\alpha)^{-1}$. Thus, 
the scale invariant solutions should be of the following form

$$
u(t, x)=\frac{1}{t^{d \lambda}} \Phi\left(\frac{x}{t^{\lambda}}\right) \quad \text { with } \quad \lambda=\frac{1}{d(m-1)+\alpha},
$$

for some function $\Phi: \mathbb{R}^{d} \rightarrow \mathbb{R}$ satisfying the following nonlocal "elliptic type" equation

$$
-\lambda \nabla \cdot(y \Phi)=\nabla \cdot\left(|\Phi| \nabla^{\alpha-1}\left(|\Phi|^{m-2} \Phi\right)\right) \quad \text { where } \quad y=\frac{x}{t^{\lambda}}
$$

Theorem 2.2 (Self-similar solutions). Let $\alpha \in(0,2], m>1$. Consider the function $\Phi_{\alpha, m}: \mathbb{R}^{d} \rightarrow \mathbb{R}$ defined as

$$
\Phi_{\alpha, m}(y)=\left(k_{\alpha, d}\left(1-|y|^{2}\right)_{+}^{\frac{\alpha}{2}}\right)^{\frac{1}{m-1}}
$$

with the constant

$$
k_{\alpha, d}=\frac{d \Gamma\left(\frac{d}{2}\right)}{(d(m-1)+\alpha) 2^{\alpha} \Gamma\left(1+\frac{\alpha}{2}\right) \Gamma\left(\frac{d+\alpha}{2}\right)} .
$$

Then, the function $u:(0, \infty) \times \mathbb{R}^{d} \rightarrow \mathbb{R}^{+}$defined by (2.1) with $\Phi=\Phi_{\alpha, m}$ is a weak solution of (1.1) in the sense of Definition 2.1 in $Q_{\eta, T} \equiv(\eta, T) \times \mathbb{R}^{d}$ for every $0<\eta<T<\infty$. Moreover, $u(t, x)$ satisfies the equation in the pointwise sense for $|x| \neq t^{\frac{1}{d(m-1)+\alpha}}$, and is $\min \left\{\frac{\alpha}{2(m-1)}, 1\right\}$-Hölder continuous at the interface $|x|=t^{\frac{1}{d(m-1)+\alpha}}$.

Remark 2.3. When $\alpha=2$ in expression (2.4) below, we recover the classical Barenblatt-Kompaneets-PattleZel'dovich solutions of the porous medium equation (1.4), see for instance [28, 27.

Remark 2.4. For each $M \in(0, \infty)$ we can find a nonnegative self-similar solution $u$ with prescribed mass $M \equiv \int u(t, x) \mathrm{d} x$ (which is conserved in time) by a suitable scaling of the profile $\Phi_{\alpha, m}$. Indeed, this self-similar solution is given by the formula

$$
u(t, x)=t^{-\frac{d}{d(m-1)+\alpha}}\left(k_{\alpha, d}\left(R^{2}-\left|x t^{-\frac{1}{d(m-1)+\alpha}}\right|^{2}\right)_{+}^{\frac{\alpha}{2}}\right)^{\frac{1}{m-1}},
$$

where, for each $M>0$, there exists a unique $R>0$ such that $\int u(t, x) \mathrm{d} x=M$.

Remark 2.5. Self-similar solutions of equation (1.6) (which is a particular case of equation (1.1) have been proved to exist in [7] by studying the following obstacle problem for the fractional Laplacian. For $\alpha \in(0,2)$ and $\Psi(y)=C-a|y|^{2}$ where $a=a(d, \alpha)$ and $C>0$, one looks for a function $P=P(y)$ with the following properties:

$$
P \geq \Psi, \quad(-\Delta)^{\frac{\alpha}{2}} P \geq 0, \quad \text { and } \quad \text { either } \quad P=\Psi \quad \text { or } \quad(-\Delta)^{\frac{\alpha}{2}} P=0 .
$$

The novelty of our approach is that we exhibit the explicit self-similar profile $\Phi_{\alpha, 2}$ defined in (2.3) and, consequently, the explicit solution of this obstacle problem: $P(y)=\mathcal{I}_{\alpha}\left(\Phi_{\alpha, 2}\right)\left(\frac{y}{R}\right)$, where $\mathcal{I}_{\alpha}=(-\Delta)^{-\frac{\alpha}{2}}$ is the Riesz potential and $R>0$ is a suitable constant.

Next, we prove the existence of weak solutions to the initial value problem (1.1)-(1.2).

Theorem 2.6 (Existence and decay of $L^{p}$-norms). Let $\alpha \in(0,2)$ and

$$
\begin{cases}m>1+\frac{1-\alpha}{d} & \text { if } \alpha \in(0,1] \\ m>3-\frac{2}{\alpha} & \text { if } \alpha \in(1,2) .\end{cases}
$$


Given $u_{0} \in L^{1}\left(\mathbb{R}^{d}\right)$, there exists a global-in-time weak solution u of the Cauchy problem (1.1)-(1.2). Moreover,

$$
\int u(t, x) \mathrm{d} x=\int u_{0}(x) \mathrm{d} x
$$

and

$$
\|u(t)\|_{p} \leq C(d, \alpha, m)\left\|u_{0}\right\|_{1}^{\frac{d(m-1) / p+\alpha}{d(m-1)+\alpha}} t^{-\frac{d}{d(m-1)+\alpha}}\left(1-\frac{1}{p}\right) \quad \text { for all } \quad t>0
$$

holds with the constant $C(d, \alpha, m)$ independent of $p$ and $u_{0}$.

The solution $u$ is nonnegative if the initial condition $u_{0}$ is so. If $u_{0} \in L^{p}\left(\mathbb{R}^{d}\right)$ for some $p \in[1, \infty]$, then

$$
\|u(t)\|_{p} \leq\left\|u_{0}\right\|_{p}
$$

holds for all $t>0$.

Remark 2.7. Estimates (2.6) are sharp since the decay in Theorem 2.6 corresponds exactly to that for self-similar solutions constructed in Theorem 2.2 Moreover, for $\alpha=2$, they are similar to those for degenerate partial differential equations like the porous medium equation (showing the regularization effect on the $L^{p}$-norms of solutions); see, e.g., [28, [8, Ch. 2].

Remark 2.8. After proving those hypercontractivity estimates in [3], we learned that a similar result is obtained in [6], however, for a less general model: $\alpha \in(0,1), m=2$, and nonnegative $u_{0}$. Moreover, analogous decay estimates for another fractional porous medium equation of the form $\partial_{t} u+(-\Delta)^{\frac{\alpha}{2}}\left(|u|^{m-1} u\right)=0$ were proved recently in [1].

Compared with the methods used in [5], we propose an alternative strategy of the proof of the existence of solutions. In this paper, we consider approximating solutions $u=u^{\delta, \varepsilon}$ of the equation

$$
\partial_{t} u=\delta \Delta u+\nabla \cdot\left(|u| \nabla^{\alpha-1} G_{\varepsilon}(u)\right)
$$

considered in the whole space $\mathbb{R}^{d}$, where $G_{\varepsilon}(u)$ is a sufficiently smooth approximation of $u|u|^{m-2}$, and then we pass to the limit with the parameters $\varepsilon \searrow 0$, and $\delta \searrow 0$. Solutions of the approximating equation exist because the parabolic regularization term $\delta \Delta u$ is strong enough to regularize equation (1.1) when $0<\alpha<2$, but of course not for $\alpha=2$. Our approach resembles the approach to the one-dimensional model achieved in [4, Sec. 4 and 5] via viscosity solutions.

\section{Preliminaries}

In this section, we collect known results that we will used in proofs of the main theorems.

Bessel and hypergeometric functions. Bessel functions of order $\nu$ are denoted by $J_{\nu}(z)$, and they behave for small and large values of the (complex) variable $z$ like

$$
\begin{aligned}
& J_{\nu}(z) \sim \quad \frac{1}{\Gamma(\nu+1)}\left(\frac{z}{2}\right)^{\nu} \quad \text { as } z \rightarrow 0, \\
& J_{\nu}(z) \sim\left(\frac{2}{\pi}\right)^{\frac{1}{2}} \cos \left(z-\frac{\nu \pi}{2}-\frac{\pi}{4}\right) z^{-\frac{1}{2}} \quad \text { as } \quad|z| \rightarrow \infty,
\end{aligned}
$$


where for functions $f, g$, the relation $f \sim g$ means that $\frac{f}{g} \rightarrow 1$. For the proofs of those properties of $J_{\nu}$, the reader is referred to, e.g., 30.

The hypergeometric function, denoted by ${ }_{2} F_{1}(a, b ; c ; z)$, is defined for complex numbers $a, b, c$ and $z$ as the sum of the series

$$
{ }_{2} F_{1}(a, b ; c ; z)=\sum_{n=0}^{\infty} \frac{(a)_{n}(b)_{n}}{(c)_{n} n !} z^{n} \quad \text { for }|z|<1,
$$

where $(a)_{n} \equiv \frac{\Gamma(a+n)}{\Gamma(a)}$, and $\Gamma$ denotes the Euler Gamma function. This series is absolutely convergent in the open unit disc and also on the circle $|z|=1$ if $\Re(a+b-c)<0$.

It is known [19, p. 39] that when $b=-n$ is a negative integer, ${ }_{2} F_{1}(a,-n ; c ; z)$ is a polynomial function of degree $n$. In particular, we have

$$
{ }_{2} F_{1}(a,-1 ; c ; z)=1-\frac{a}{c} z .
$$

We will also use the following differentiation formula [19, p. 41]

$$
\frac{d}{d z}\left({ }_{2} F_{1}(a, b ; c ; z)\right)=\frac{a b}{c}{ }_{2} F_{1}(a+1, b+1 ; c+1 ; z) .
$$

The Weber-Schafheitlin integral. If $0<b<a$ and if integral (3.3) below is convergent, then the following identity holds true

$$
\int_{0}^{\infty} t^{-\lambda} J_{\mu}(a t) J_{\nu}(b t) \mathrm{d} t=\frac{b^{\nu} 2^{-\lambda} a^{\lambda-\nu-1} \Gamma\left(\frac{\nu+\mu-\lambda+1}{2}\right)}{\Gamma\left(\frac{-\nu+\mu+\lambda+1}{2}\right) \Gamma(1+\nu)}{ }_{2} F_{1}\left(\frac{\nu+\mu-\lambda+1}{2}, \frac{\nu-\mu-\lambda+1}{2} ; \nu+1 ; \frac{b^{2}}{a^{2}}\right) .
$$

According to Watson, [30, pp. 401-403], this result was obtained by Sonine and Schafheitlin. However, it is usually referred to as the Weber-Schafheitlin discontinuous integral since there occurs a discontinuity for $a=b$.

The Stroock-Varopoulos inequality. We next recall the the Stroock-Varopoulos inequality, see [18, Theorem 2.1 and Condition (1.7)] for a proof.

Proposition 3.1. For $\alpha \in(0,2], w \in \mathcal{C}_{c}^{\infty}\left(\mathbb{R}^{d}\right)$ and $q>1$, the following inequality holds true

$$
\int \operatorname{sgn} w|w|^{q-1}(-\Delta)^{\frac{\alpha}{2}} w \mathrm{~d} x \geq \frac{4(q-1)}{q^{2}} \int\left|\nabla^{\frac{\alpha}{2}}\left(\operatorname{sgn} w|w|^{\frac{q}{2}}\right)\right|^{2} \mathrm{~d} x \geq\left.\left.\frac{4(q-1)}{q^{2}} \int\left|\nabla^{\frac{\alpha}{2}}\right| w\right|^{\frac{q}{2}}\right|^{2} \mathrm{~d} x .
$$

Fractional order Sobolev spaces. The fractional order Sobolev spaces are defined as

$$
H^{s, p}\left(\mathbb{R}^{d}\right)=\left\{v \in L^{p}\left(\mathbb{R}^{d}\right): \nabla^{s} v \in L^{p}\left(\mathbb{R}^{d}\right)\right\}=\left\{v \in L^{p}\left(\mathbb{R}^{d}\right):(I-\Delta)^{\frac{s}{2}} v \in L^{p}\left(\mathbb{R}^{d}\right)\right\},
$$

here with $p \in(1, \infty)$, supplemented with the usual norm denoted by $\|\cdot\|_{H^{s, p}}$, and we refer the reader to the books [25, 26] for properties of those spaces. In particular for $s=\alpha-1$ with $\alpha \in(1,2)$, the following well-known continuous embedding will be used repeatedly

$$
H^{\alpha-1, p}\left(\mathbb{R}^{d}\right) \subset L^{\infty}\left(\mathbb{R}^{d}\right) \quad \text { provided } \quad p>\frac{d}{\alpha-1} \quad(>1) .
$$

We also recall the fractional integration theorem [23, Ch. V, 11.2$]$ : the Riesz potential $\mathcal{I}_{s}=(-\Delta)^{-\frac{s}{2}}$ satisfies

$$
\left\|\mathcal{I}_{s} u\right\|_{q} \leq C(p, q, s)\|u\|_{p}
$$

for all $s \in(0, d)$ and $p, q \in(1, \infty)$ satisfying $\frac{1}{q}=\frac{1}{p}-\frac{s}{d}$. 
Some functional inequalities. We will use the following Nash inequality

$$
\|v\|_{2}^{2\left(1+\frac{\alpha}{d}\right)} \leq C_{N}\left\|\nabla^{\frac{\alpha}{2}} v\right\|_{2}^{2}\|v\|_{1}^{\frac{2 \alpha}{d}}
$$

valid for all functions $v \in L^{1}\left(\mathbb{R}^{d}\right)$, such that $\nabla^{\frac{\alpha}{2}} v \in L^{2}\left(\mathbb{R}^{d}\right)$, and with a constant $C_{N}=C(d, \alpha)>0$. The proof of (3.7) for $d=1$ can be found in, e.g., [16, Lemma 2.2], and this extends easily to the general case $d \geq 1$.

Moreover, we will use the following Gagliardo-Nirenberg type inequality

Lemma 3.2. For $p>1$ and $p \geq m-1$, the inequality

$$
\|u\|_{p}^{a} \leq C_{N}\left\|\nabla^{\frac{\alpha}{2}}|u|^{\frac{r}{2}}\right\|_{2}^{2}\|u\|_{1}^{b}
$$

holds with

$$
a=\frac{p}{p-1} \frac{d(r-1)+\alpha}{d}, \quad b=a-r=\frac{d(m-1)+p \alpha}{d(p-1)}, \quad r=p+m-1 .
$$

Proof. This inequality is a consequence of the Nash inequality (3.7) written for $v=|u|^{\frac{r}{2}}$, i.e.

$$
\|u\|_{r}^{r\left(1+\frac{\alpha}{d}\right)} \leq C_{N}\left\|\nabla^{\frac{\alpha}{2}}|u|^{\frac{r}{2}}\right\|_{2}^{2}\|u\|_{\frac{r}{2}}^{\frac{r \alpha}{d}},
$$

and two Hölder inequalities

$$
\|u\|_{p} \leq\|u\|_{r}^{\gamma}\|u\|_{1}^{1-\gamma} \quad \text { with } \quad \gamma=\left(\frac{p-1}{r-1}\right) \frac{r}{p}
$$

and

$$
\|u\|_{\frac{r}{2}} \leq\|u\|_{p}^{\delta}\|u\|_{1}^{1-\delta} \quad \text { with } \quad \delta=\left(\frac{r-2}{p-1}\right) \frac{p}{r} .
$$

Combining the above three inequalities, we get (3.8).

\section{Proof of Theorem 2.2}

This section is devoted to the study of nonnegative self-similar solutions for (1.1) with $m>1$. As explained above, this problem reduces to a study of the elliptic-like equation (2.2) which for nonnegative $\Phi$ takes the form

$$
-\lambda y \Phi=\Phi \nabla^{\alpha-1}\left(\Phi^{m-1}\right) .
$$

Moreover, since we want to construct compactly supported solutions, we are interested in solutions $\Phi$ vanishing outside the unit ball $B_{1}$. This is the reason why we consider the Dirichlet problem

$$
\begin{aligned}
-\lambda y=\nabla^{\alpha-1}\left(\Phi^{m-1}\right) & \text { in } \quad B_{1}, \\
\Phi=0 & \text { in } \quad \mathbb{R}^{d} \backslash B_{1} .
\end{aligned}
$$

It is well known that, in the case of nonlocal operators (such as $\nabla^{\alpha-1}$ ), the homogeneous Dirichlet condition should be understood in the form $\Phi \equiv 0$ outside the domain $B_{1}$, and not only $\Phi=0$ on the boundary $\partial B_{1}$. The reader is referred to, e.g., 2] for more explanations.

We claim that the proof of Theorem 2.2 reduces to the following key computation. Here, ${ }_{2} F_{1}$ denotes the classical hypergeometric function defined in Section 3 
Lemma 4.1. For all $\beta \in(0,2), \beta<d$, and $\gamma>0$, we have

$$
\mathcal{I}_{\beta}\left(\left(1-|y|^{2}\right)_{+}^{\frac{\gamma}{2}}\right)= \begin{cases}C_{\gamma, \beta, d} \times{ }_{2} F_{1}\left(\frac{d-\beta}{2},-\frac{\gamma+\beta}{2} ; \frac{d}{2} ;|y|^{2}\right) & \text { for }|y| \leq 1, \\ \tilde{C}_{\gamma, \beta, d}|y|^{\beta-d} \times{ }_{2} F_{1}\left(\frac{d-\beta}{2}, \frac{2-\beta}{2} ; \frac{d+\gamma}{2} ; \frac{1}{|y|^{2}}\right) & \text { for }|y|>1,\end{cases}
$$

with $C_{\gamma, \beta, d}=2^{-\beta} \frac{\Gamma\left(\frac{\gamma}{2}+1\right) \Gamma\left(\frac{d-\beta}{2}\right)}{\Gamma\left(\frac{d}{2}\right) \Gamma\left(\frac{\beta+\gamma}{2}+1\right)}$ and $\tilde{C}_{\gamma, \beta, d}=2^{-\beta} \frac{\Gamma\left(\frac{\gamma}{2}+1\right) \Gamma\left(\frac{d-\beta}{2}\right)}{\Gamma\left(\frac{d}{4}\right) \Gamma\left(\frac{d+\gamma}{2}+1\right)}$.

The proof of this lemma is postponed to the end of this section.

The following corollary is an immediate consequence of Lemma 4.1 with $2-\beta=\alpha=\gamma$, of the property of ${ }_{2} F_{1}$ formulated in (3.1), and of the identity $(-\Delta)^{\frac{\alpha}{2}}=(-\Delta) \mathcal{I}_{2-\alpha}$. It has an important probabilistic interpretation, and recently, related results and generalizations have been proved in [13].

Corollary 4.2 (Getoor [14, Th. 5.2]). For all $\alpha \in(0,2]$, the identity

$$
K_{\alpha, d}(-\Delta)^{\frac{\alpha}{2}}\left(1-|y|^{2}\right)_{+}^{\frac{\alpha}{2}}=1 \text { in } B_{1}
$$

holds true with the constant $K_{\alpha, d}=\frac{\Gamma\left(\frac{d}{2}\right)}{2^{\alpha} \Gamma\left(1+\frac{\alpha}{2}\right) \Gamma\left(\frac{d+\alpha}{2}\right)}$.

Before proving Lemma 4.1, we first use it to derive Theorem 2.2

Proof of Theorem 2.2. We check that $u(t, x)=t^{-d \lambda} \Phi_{\alpha, m}\left(t^{-\lambda} x\right)$ is a weak solution of (1.1) in the sense of Definition 2.1. First, $u \in L^{1}\left(Q_{T}\right)$ if and only if $\Phi_{\alpha, m} \in L^{1}\left(\mathbb{R}^{d}\right)$, which is obviously true. For later use, it is convenient to introduce the function $\Phi_{\alpha}(u)=\left(1-|y|^{2}\right)_{+}^{\frac{\alpha}{2}}$, so that $k_{\alpha, d} \Phi_{\alpha}=\Phi_{\alpha, m}^{m-1}$.

The fact that, for all $\eta, T$ such that $0<\eta<T, u \nabla^{\alpha-1}\left(u^{m-1}\right)$ and $\nabla^{\alpha-1}\left(u^{m-1}\right)$ are locally integrable in $(\eta, T) \times \mathbb{R}^{d}$ follows from

$$
\mathcal{I}_{2-\alpha}\left(\Phi_{\alpha}\right) \in H_{\mathrm{loc}}^{1,1}\left(\mathbb{R}^{d}\right)
$$

which we prove by computing $\mathcal{I}_{2-\alpha}\left(\Phi_{\alpha}\right)$. In order to do so, we first assume that $\alpha>2-d$, and we apply Lemma 4.1 with $\gamma=\alpha \in(0,2)$ and $\beta=2-\alpha$, we use equation (3.1), and we get

$$
\mathcal{I}_{2-\alpha}\left(\Phi_{\alpha}\right)(y)= \begin{cases}C_{\alpha, 2-\alpha, d}\left(1-\frac{d+\alpha-2}{d}|y|^{2}\right) & \text { if }|y| \leq 1, \\ \tilde{C}_{\alpha, 2-\alpha, d}|y|^{2-(d+\alpha)}{ }_{2} F_{1}\left(\frac{d+\alpha}{2}-1, \frac{\alpha}{2} ; \frac{d+\alpha}{2} ; \frac{1}{|y|^{2}}\right) & \text { if }|y|>1\end{cases}
$$

The right-hand side of equation (4.3) defines a locally integrable function because

$$
a+b-c=\left(\frac{d+\alpha}{2}-1\right)+\frac{\alpha}{2}-\frac{d+\alpha}{2}<0 .
$$

We then deduce that

$$
\nabla^{\alpha-1}\left(\Phi_{\alpha}\right)(y)=\nabla \mathcal{I}_{2-\alpha}\left(\Phi_{\alpha}\right)(y)=-\frac{\lambda}{k_{\alpha, d}} y \text { for } y \in B_{1}
$$

Note also that $\nabla^{\alpha-1}\left(\Phi_{\alpha}\right)$ can be computed outside $B_{1}$ thanks to the differentiation formula (3.2).

We now remark that

$$
\Phi_{\alpha, m}(y)\left(\nabla^{\alpha-1} \Phi_{\alpha, m}^{m-1}\right)(y)=-\lambda y \Phi_{\alpha, m}(y) \text { for all } y \in \mathbb{R}^{d}
$$


which is in $L^{1}\left(\mathbb{R}^{d}\right)$. Moreover, the following equalities hold true in the sense of distributions in $Q_{T}$,

$$
\begin{aligned}
\partial_{t} u(t, x) & =-\lambda t^{-d \lambda-1} \nabla_{y} \cdot\left(y \Phi_{\alpha, m}\right)\left(t^{-\lambda} x\right) \\
\nabla_{x} \cdot\left(u \nabla^{\alpha-1}\left(|u|^{m-1}\right)\right)(t, x) & =t^{-d \lambda-1} \nabla_{y} \cdot\left(\Phi_{\alpha, m} \nabla^{\alpha-1} \Phi_{\alpha, m}^{m-1}\right)\left(t^{-\lambda} x\right) .
\end{aligned}
$$

This allows us to conclude that $u$ is indeed a weak solution of (1.1) in $(\eta, T) \times \mathbb{R}^{d}$ for all $0<\eta<T<\infty$ if $\alpha>2-d$.

Assume now that $0<\alpha \leq 2-d$, which means that $d=1$ and $\alpha \leq 1$. The critical case $\alpha=1$ can be obtained by passing to the limit as $\alpha \searrow 1$; indeed, the constants $C_{\alpha, 2-\alpha, d}(d+\alpha-2)$ and $\tilde{C}_{\alpha, 2-\alpha, d}(d+\alpha-2)$ appearing in (4.3) simplify thanks to the relation $z \Gamma(z)=\Gamma(z+1)$. If now $\alpha<1$, we can argue as above by analytic continuation. The proof is now complete.

Now we turn to the proof of the main technical lemma.

Proof of Lemma 4.1. We first assume that $\beta \in(0, d)$ and that $\gamma>\max \{0, d-2 \beta-1\}$, and we then argue by the analytic continuation with a choice of parameters corresponding each time to ${ }_{2} F_{1}$ defined and bounded for all $|y| \leq 1$.

The Fourier transform of $\Phi_{\gamma}(y)=\left(1-|y|^{2}\right)_{+}^{\frac{\gamma}{2}}$ is expressed in terms of Bessel functions, see, e.g., [23, Ch. IV, Sec. 3]

$$
\mathcal{F}\left(\Phi_{\gamma}\right)(\xi)=2^{\frac{\gamma}{2}} \Gamma\left(\frac{\gamma}{2}+1\right) \frac{1}{|\xi|^{\frac{d+\gamma}{2}}} J_{\frac{d+\gamma}{2}}(|\xi|) .
$$

Since $\mathcal{I}_{\beta}$ is the Fourier multiplier of symbol $|\xi|^{-\beta}, \mathcal{I}_{\beta}\left(\Phi_{\gamma}\right)$ is the (inverse) Fourier transform of the following radially symmetric function

$$
2^{\frac{\gamma}{2}} \Gamma\left(\frac{\gamma}{2}+1\right) \frac{1}{|\xi|^{\frac{d+\gamma}{2}+\beta}} J_{\frac{d+\gamma}{2}}(|\xi|) .
$$

We recall that by properties of Bessel functions collected in Section 3, we have $J_{\nu}(r)=\mathcal{O}\left(r^{\nu}\right)$ as $r \rightarrow 0$ and $J_{\nu}(r)=\mathcal{O}\left(r^{-\frac{1}{2}}\right)$ as $r \rightarrow \infty$. We see that the previous function is integrable since $\beta<d$ and $d<\gamma+2 \beta+1$. Thanks to [24, Th. 3.3], we get

$$
\begin{aligned}
\mathcal{I}_{\beta}\left(\Phi_{\gamma}\right)(y) & =2^{\frac{\gamma}{2}} \Gamma\left(\frac{\gamma}{2}+1\right)|y|^{1-\frac{d}{2}} \int_{0}^{\infty} \frac{1}{t^{\frac{d+\gamma}{2}+\beta}} J_{\frac{d+\gamma}{2}}(t) t^{\frac{d}{2}} J_{\frac{d}{2}-1}(t|y|) \mathrm{d} t \\
& =2^{\frac{\gamma}{2}} \Gamma\left(\frac{\gamma}{2}+1\right)|y|^{1-\frac{d}{2}} \int_{0}^{\infty} t^{-\left(\frac{\gamma}{2}+\beta\right)} J_{\frac{d+\gamma}{2}}(t) J_{\frac{d}{2}-1}(t|y|) \mathrm{d} t .
\end{aligned}
$$

We obtain (4.2) applying (3.3) with the following choice of parameters:

- if $|y| \leq 1$, we put $\lambda=\frac{\gamma}{2}+\beta, \mu=\frac{d+\gamma}{2}, \nu=\frac{d}{2}-1, a=1$ and $b=|y|$,

- if $|y|>1$, we put $\lambda=\frac{\gamma}{2}+\beta, \mu=\frac{d}{2}-1, \nu=\frac{d+\gamma}{2}, a=|y|$ and $b=1$. 


\section{A REGULARIZED PROBLEM}

In order to construct weak solutions of (1.1) for general initial data, we first consider the following regularized problem

$$
\partial_{t} u=\delta \Delta u+\nabla \cdot\left(|u| \nabla^{\alpha-1}(G(u))\right), \quad u(0, x)=u_{0}(x),
$$

where $G: \mathbb{R} \rightarrow \mathbb{R}$ satisfies

$$
\left\{\begin{array}{l}
G \text { differentiable and increasing } \\
G(0)=G^{\prime}(0)=0 \\
G^{\prime} \text { locally Lipschitz continuous. }
\end{array}\right.
$$

Remark that for $m \geq 3$ or $m=2$, the function $G(u)=|u|^{m-2} u$ satisfies (5.2). For $m \in(1,3)$, we consider the following approximation $G=G_{\varepsilon}$ of $|u|^{m-2} u$

$$
G_{\varepsilon}(u)=\operatorname{sgn} u\left(\left(u^{2}+\varepsilon^{2}\right)^{\frac{m-1}{2}}-\varepsilon^{m-1}\right)
$$

with $\varepsilon>0$. The following theorem holds true for a general function $G$ satisfying (5.2).

Theorem 5.1 (Existence of solutions to the regularized problem). Let $\delta>0$ and assume that $G$ is an arbitrary function satisfying (5.2). Moreover, assume

$$
u_{0} \in \begin{cases}L^{1}\left(\mathbb{R}^{d}\right) \cap L^{\infty}\left(\mathbb{R}^{d}\right) & \text { if } \quad \alpha \in(0,1], \\ L^{1}\left(\mathbb{R}^{d}\right) \cap\left(\cap_{p>p_{\alpha}} H^{\alpha-1, p}\left(\mathbb{R}^{d}\right)\right) & \text { if } \quad \alpha \in(1,2),\end{cases}
$$

with $p_{\alpha}=\frac{d}{\alpha-1}>1$. There exists a unique function $u$ in the space

$$
u \in \begin{cases}\mathcal{C}\left([0, \infty), L^{1}\left(\mathbb{R}^{d}\right) \cap L^{\infty}\left(\mathbb{R}^{d}\right)\right) & \text { if } \alpha \in(0,1], \\ \cap_{p>p_{\alpha}} \mathcal{C}\left([0, \infty), L^{1}\left(\mathbb{R}^{d}\right) \cap H^{\alpha-1, p}\left(\mathbb{R}^{d}\right)\right) & \text { if } \alpha \in(1,2),\end{cases}
$$

satisfying problem (5.1) in the usual weak sense

$$
\iint\left(u \partial_{t} \varphi-|u| \nabla^{\alpha-1}(G(u)) \cdot \nabla \varphi-\delta \nabla u \cdot \nabla \varphi\right) \mathrm{d} t \mathrm{~d} x=0
$$

for all $\varphi \in \mathcal{C}_{c}^{\infty}\left(Q_{T}\right)$.

Moreover, $u(t, x)$ is nonnegative if the initial condition $u_{0}$ is so, and for all $t>0$ and $q \in[1, \infty]$ we have

$$
\int u(t, x) \mathrm{d} x=\int u_{0}(x) \mathrm{d} x \quad \text { and } \quad\|u(t)\|_{q} \leq\left\|u_{0}\right\|_{q} .
$$

Local-in-time existence of mild solutions.

Proposition 5.2. Let $p>p_{\alpha}=\frac{d}{\alpha-1}$. There exists $T>0$ depending only on $u_{0}$, and a function $u$ in the space

$$
u \in \begin{cases}\mathcal{C}\left([0, T), L^{1}\left(\mathbb{R}^{d}\right) \cap L^{\infty}\left(\mathbb{R}^{d}\right)\right) & \text { if } \alpha \in(0,1] \\ \mathcal{C}\left([0, T), L^{1}\left(\mathbb{R}^{d}\right) \cap H^{\alpha-1, p}\left(\mathbb{R}^{d}\right)\right) & \text { if } \alpha \in(1,2)\end{cases}
$$

such that

$$
u(t)=\mathrm{e}^{\delta t \Delta} u_{0}+\int_{0}^{t} \nabla \mathrm{e}^{\delta(t-s) \Delta} \cdot \Psi(u(s)) \mathrm{d} s \quad \text { with } \quad \Psi(u)=|u| \nabla^{\alpha-1} G(u),
$$

in $\left.\mathcal{C}[0, T], L^{1}\left(\mathbb{R}^{d}\right) \cap L^{\infty}\left(\mathbb{R}^{d}\right)\right)$ where $\mathrm{e}^{t \Delta}$ denotes the heat semigroup. 
Remark 5.3. We identify the heat semigroup $\mathrm{e}^{t \Delta}$ and its kernel $(4 \pi t)^{-\frac{d}{2}} \exp \left(-\frac{|x|^{2}}{4 t}\right)$. We will use the following classical fact

$$
\left\|\nabla^{\beta} \mathrm{e}^{\delta t \Delta} v\right\|_{p} \leq C(p, r, \beta, \delta) t^{-\frac{d}{2}\left(\frac{1}{r}-\frac{1}{p}\right)-\frac{\beta}{2}}\|v\|_{r}
$$

with $1 \leq r \leq p \leq \infty$, and $\beta \in[1,2)$.

Now we turn to the proof of Proposition 5.2 .

Proof of Proposition 5.2. We look for a solution $u \in \mathcal{C}([0, T], X)$ as a fixed point of the map

$$
\mathcal{T}: u \mapsto \mathrm{e}^{\delta t \Delta} u_{0}+\int_{0}^{t} \nabla \mathrm{e}^{\delta(t-s) \Delta} \cdot \Psi(u(s)) \mathrm{d} s,
$$

where $X$ is chosen as follows

$$
X= \begin{cases}L^{1}\left(\mathbb{R}^{d}\right) \cap L^{\infty}\left(\mathbb{R}^{d}\right) & \text { if } \alpha \in(0,1] \\ L^{1}\left(\mathbb{R}^{d}\right) \cap H^{\alpha-1, p}\left(\mathbb{R}^{d}\right) & \text { if } \alpha \in(1,2) .\end{cases}
$$

The associated norms are $\|u\|_{1}+\|u\|_{Y}$ with $Y=L^{\infty}\left(\mathbb{R}^{d}\right)$ and $Y=H^{\alpha-1, p}\left(\mathbb{R}^{d}\right)$, respectively. We show that $\mathcal{T}$ has a fixed point by the Banach contraction principle as soon as $T=T\left(\left\|u_{0}\right\|_{X}\right)>0$ is sufficiently small.

In both cases, it is enough to prove the following lemma.

Lemma 5.4. For all $T \in(0,1)$, the operator $\mathcal{T}$ maps $\mathcal{C}([0, T], X)$ into itself. Moreover, there exist $C>0$ and $\gamma>0$ such that for all $u, v \in \bar{B}(0, R) \subset \mathcal{C}([0, T], X)$,

$$
\|\mathcal{T}(u)-\mathcal{T}(v)\|_{\mathcal{C}([0, T], X)} \leq C_{1}(R) T^{\gamma}\|u-v\|_{\mathcal{C}([0, T], X)}
$$

where $C_{1}(R)$ is a constant which also depends on $\alpha, d, \varepsilon, m, \delta$ (and on $p$ if $\left.\alpha \in(1,2)\right)$.

Indeed, once this lemma is proved, we first derive

$$
\|\mathcal{T}(u)\|_{\mathcal{C}([0, T], X)} \leq\left\|u_{0}\right\|_{X}+R C_{1}(R) T^{\gamma}
$$

by choosing $v=0$ in (5.11) and using estimate (5.9). Now it is enough to choose $R=2\left\|u_{0}\right\|_{X}$ and $T>0$ such that $C_{1}(R) T^{\gamma} \leq \frac{1}{2}$ in order to ensure that $\mathcal{T}$ maps $\bar{B}(0, R)$ into itself, and is a contraction.

The case $\alpha \in(0,1]$. In order to get estimate (5.11), we first write

$$
\mathcal{T}(u)(t)-\mathcal{T}(v)(t)=\int_{0}^{t} \nabla \mathrm{e}^{\delta(t-s) \Delta} \cdot(\Psi(u)-\Psi(v))(s) \mathrm{d} s,
$$

and the difference of $\Psi$ 's is represented as

$$
\Psi(u)-\Psi(v)=(|u|-|v|) \nabla^{\alpha-1} G(u)+|v| \nabla^{\alpha-1}(G(u)-G(v)) .
$$

Lemma 5.5. For every $\alpha \in(0,1]$ and $p \in(1, \infty)$ there exists a constant $C(p, \alpha)>0$ such that for all $u \in$ $L^{\infty}\left(\mathbb{R}^{d}\right) \cap L^{p}\left(\mathbb{R}^{d}\right)$ the following inequality

$$
\left\|\nabla^{\alpha-1}(G(u)-G(v))\right\|_{q} \leq C(p, \alpha)\left(\sup _{|z| \leq\|u\|_{\infty}+\|v\|_{\infty}} G^{\prime}(z)\right)\|u-v\|_{p}
$$

holds true with $\frac{1}{q}=\frac{1}{p}-\frac{1-\alpha}{d}$. 
Proof. Since, for $\alpha \in(0,1]$, we have $\nabla^{\alpha-1}=\nabla^{0} \mathcal{I}_{1-\alpha}$, where the components of $\nabla^{0}$ are the Riesz transforms (which are bounded operators on $L^{p}\left(\mathbb{R}^{d}\right)$ for each $p \in(1, \infty)$ ), we obtain (5.15) from estimate (3.6) as follows

$$
\left\|\nabla^{\alpha-1}(G(u)-G(v))\right\|_{q} \leq\left\|\mathcal{I}_{1-\alpha}(G(u)-G(v))\right\|_{q} \leq C(p, \alpha)\|G(u)-G(v)\|_{p} \leq C\|u-v\|_{p} .
$$

Now, we come back to the proof of (5.11) with $X=L^{1}\left(\mathbb{R}^{d}\right) \cap L^{\infty}\left(\mathbb{R}^{d}\right)$, First, for all $u, v \in \bar{B}(0, R) \subset X$ and some $q \in(1, \infty)$,

$$
\begin{aligned}
\|\Psi(u)-\Psi(v)\|_{1} & \leq C\|u-v\|_{q^{*}}\left\|\nabla^{\alpha-1} G(u)\right\|_{q}+C\|v\|_{q^{*}}\left\|\nabla^{\alpha-1}(G(u)-G(v))\right\|_{q} \\
& \leq C\|u-v\|_{q^{*}} G^{\prime}\left(\|u\|_{\infty}\right)\|u\|_{p}+C\|v\|_{q^{*}} G^{\prime}\left(\|u\|_{\infty}+\|v\|_{\infty}\right)\|u-v\|_{p} \\
& \leq C_{0}(R)\|u-v\|_{X}
\end{aligned}
$$

with $C_{0}(R)=C\left(\alpha, d, q, R G^{\prime}(2 R)\right)$ and $\frac{1}{q}+\frac{1}{q^{*}}=1$ and $\frac{1}{q}=\frac{1}{p}-\frac{1-\alpha}{d}$. We used estimate (5.15) twice to get the second line in (5.16), and the inequality $\|u\|_{r} \leq\|u\|_{X}$ which is valid for all $r \in[1, \infty]$ to obtain the last one.

The estimate of the second norm in $X$ is obtained similarly: for all $u, v \in \bar{B}(0, R) \subset X$ and some $q \in(1, \infty)$,

$$
\|\Psi(u)-\Psi(v)\|_{q} \leq\|u-v\|_{\infty}\left\|\nabla^{\alpha-1} G(u)\right\|_{q}+\|v\|_{\infty}\left\|\nabla^{\alpha-1}(G(u)-G(v))\right\|_{q} \leq C_{0}(R)\|u-v\|_{X} .
$$

Now, we apply inequalities (5.9) with $\beta=1,\langle p, r\rangle=\langle 1,1\rangle$ and $\langle p, r\rangle=\langle\infty, q\rangle$, respectively, and the estimates (5.16), (5.17) yield

$$
\begin{array}{r}
\|\mathcal{T}(u)-\mathcal{T}(v)\|_{\mathcal{C}\left([0, T], L^{1}\left(\mathbb{R}^{d}\right)\right)} \leq C C_{0}(R) T^{\frac{1}{2}}\|u-v\|_{\mathcal{C}[0, T], X)}, \\
\|\mathcal{T}(u)-\mathcal{T}(v)\|_{\mathcal{C}\left([0, T], L^{\infty}\left(\mathbb{R}^{d}\right)\right)} \leq C C_{0}(R) T^{\frac{1}{2}-\frac{d}{2} \frac{1}{q^{*}}}\|u-v\|_{\mathcal{C}[0, T], X)} .
\end{array}
$$

Combining (5.13), (5.14), (5.18) and (5.19), we thus get (5.11) for $\alpha \in(0,1]$, with $\gamma=\frac{1}{2}-\frac{d}{2} \frac{1}{q^{*}}$, now with a new constant $C_{1}(R)=C\left(\alpha, d, q^{*}, R G^{\prime}(2 R)\right)$, where we have chosen $q^{*}>d$ to ensure $\frac{d}{2} \frac{1}{q^{*}}<\frac{1}{2}$.

As far as the continuity of $\mathcal{T}(u)$ with respect to time is concerned, it is enough to study

$$
\mathcal{S}(u)=\int_{0}^{t} \nabla \mathrm{e}^{\delta(t-s) \Delta} \cdot \Psi(u(s)) \mathrm{d} s
$$

We fix $t \in[0, T]$ and write for $h$ small enough (and positive if $t=0$, negative if $t=T$ ),

$$
\mathcal{S}(u(t+h))-\mathcal{S}(u(t))=\int_{t}^{t+h} \nabla \mathrm{e}^{\delta(t+h-s) \Delta} \cdot \Psi(u(s)) \mathrm{d} s+\int_{0}^{t} \nabla \mathrm{e}^{\delta(t-s) \Delta} \cdot\left(\mathrm{e}^{\delta h \Delta} \Psi(u(s))-\Psi(u(s))\right) \mathrm{d} s .
$$

As above, use two key estimates (5.16) and (5.16) together with (5.9) (and the dominated convergence theorem) to conclude the proof of Proposition 5.2 for $\alpha \in(0,1]$.

The case $\alpha \in(1,2)$. We argue as before, using (5.13) and (5.14). We need now to state and to prove the corresponding key technical lemma.

Lemma 5.6. For $\alpha \in(1,2), p>\frac{d}{\alpha-1}$, and $u \in H^{\alpha-1, p}\left(\mathbb{R}^{d}\right)$,

$$
\|G(u)-G(v)\|_{H^{\alpha-1, p}} \leq C_{2}\left(\|u\|_{H^{\alpha-1, p}}+\|v\|_{H^{\alpha-1, p}}\right)\|u-v\|_{H^{\alpha-1, p}},
$$


where $C_{2}\left(\|u\|_{H^{\alpha-1, p}}+\|v\|_{H^{\alpha-1, p}}\right)$ depends on $\|u\|_{H^{\alpha-1, p}},\|v\|_{H^{\alpha-1, p}}$ and on the $W^{1, \infty}{ }_{-n o r m}$ of $G^{\prime}$ in the interval $\left[0,\|u\|_{\infty}+\|v\|_{\infty}\right]$

Proof. We use the classical identity

$$
G(u)-G(v)=K(u, v)(u-v) \quad \text { with } \quad K(u, v)=\int_{0}^{1} G^{\prime}(\tau u+(1-\tau) v) \mathrm{d} \tau
$$

and the Moser estimate for the product of two functions in $H^{\alpha-1, p}\left(\mathbb{R}^{d}\right)$, see, e.g., [25, Ch. 2, ineq. (0.22)], to obtain the following inequality

$$
\|G(u)-G(v)\|_{H^{\alpha-1, p}} \leq\|u-v\|_{\infty}\|K(u, v)\|_{H^{\alpha-1, p}}+\|u-v\|_{H^{\alpha-1, p}}\|K(u, v)\|_{\infty} .
$$

Moreover, we recall [25. Ch. 2, Prop. 4.1] that for every increasing locally Lipschitz function $H$ we have

$$
\|H(u)\|_{H^{\alpha-1, p}} \leq C\left|H^{\prime}\right|\left(\|u\|_{\infty}\right)\|u\|_{H^{\alpha-1, p}}
$$

for every $p \in(1, \infty)$ and $\alpha-1 \in(0,1)$. Choosing $H=G^{\prime}$, we deduce that

$$
\begin{aligned}
\|K(u, v)\|_{H^{\alpha-1, p}} & \leq \int_{0}^{1}\left\|G^{\prime}(\tau u+(1-\tau) v)\right\|_{H^{\alpha-1, p}} \mathrm{~d} \tau \\
& \leq C\left|G^{\prime \prime}\right|\left(\|u\|_{\infty}+\|v\|_{\infty}\right)\left(\|u\|_{H^{\alpha-1, p}}+\|v\|_{H^{\alpha-1, p}}\right) .
\end{aligned}
$$

Moreover, we have the trivial estimate

$$
\|K(u, v)\|_{\infty} \leq\left|G^{\prime}\right|\left(\|u\|_{\infty}+\|v\|_{\infty}\right)
$$

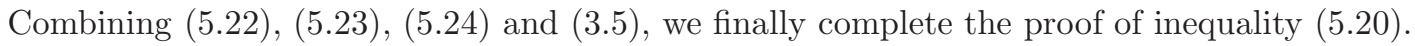

Now, we are in a position to obtain the estimate of the first component of the norm of $X$. From (5.14), we get

$$
\|\Psi(u)-\Psi(v)\|_{1} \leq C_{2}(R) R\|u-v\|_{p^{\prime}}+C_{2}(2 R)\|v\|_{p^{\prime}}\|u-v\|_{X} \leq C_{3}(R)\|u-v\|_{X}
$$

with $C_{3}(R)=C R\left(C_{2}(R)+C_{2}(2 R)\right.$ ). We used inequality (5.20) twice, as well as the fact that $\|u\|_{r} \leq C \|_{x}$ for all $r \in[1, \infty]$ and $u \in X$. From (5.25) and (5.9) with $\beta=1$ and $\langle p, r\rangle=\langle 1,1\rangle$, we get inequality (5.18) where $C_{0}(R)$ is replaced with $\tilde{C}_{0}(R)=C_{3}(R)$.

The estimate of the $H^{\alpha-1, p}$-norm is obtained analogously. First, we have

$$
\|\Psi(u)-\Psi(v)\|_{p} \leq\|u-v\|_{\infty}\left\|\nabla^{\alpha-1} G(u)\right\|_{p}+\|v\|_{\infty}\left\|\nabla^{\alpha-1}(G(u)-G(v))\right\|_{p} \leq C_{3}(R)\|u-v\|_{X}
$$

From inequalities (5.26) and (5.9) with $\beta=1$ and $\beta=\alpha$ and $\langle p, r\rangle=\langle p, p\rangle$, we get

$$
\|\mathcal{T}(u)-\mathcal{T}(v)\|_{\mathcal{C}\left([0, T], H^{\alpha-1, p}\left(\mathbb{R}^{d}\right)\right)} \leq C C_{3}(R)\left(T^{\frac{1}{2}}+T^{\frac{1}{2}-\frac{\alpha}{2}}\right)\|u-v\|_{\mathcal{C}[0, T], X)}
$$

Finally, combining (5.18) and (5.27), we complete the proof of (5.11) with $\gamma=\frac{1}{2}-\frac{\alpha}{2}$ and with some $\tilde{C}_{1}(R)$.

The time continuity of $\mathcal{T}(u)$ is proved as in the case $\alpha \in(0,1]$, and this achieves the proof of Proposition 5.2 


\section{Regularity of the solutions.}

Corollary 5.7 (Regularity of the solutions). Consider $u_{0} \in L^{1}\left(\mathbb{R}^{d}\right) \cap L^{\infty}\left(\mathbb{R}^{d}\right)$ if $\alpha \in(0,1]$ (as before), and $u_{0} \in L^{1}\left(\mathbb{R}^{d}\right) \cap\left(\cap_{p>p_{\alpha}} H^{\alpha-1, p}\left(\mathbb{R}^{d}\right)\right)$ if $\alpha \in(1,2)$ (a strengthened assumption). Then the solution constructed in Proposition 5.2 enjoys the following regularity

$$
u \in \mathcal{C}^{1}\left((0, T), L^{p}\left(\mathbb{R}^{d}\right)\right) \cap \mathcal{C}\left((0, T), H^{1, p}\left(\mathbb{R}^{d}\right)\right)
$$

for every $p \in\left(\bar{p}_{\alpha}, \infty\right)$ with

$$
\bar{p}_{\alpha}= \begin{cases}\frac{d}{d-(1-\alpha)} & \text { if } \alpha \in(0,1] \\ \frac{d}{\alpha-1} & \text { if } \alpha \in(1,2) .\end{cases}
$$

In particular, $u=u(t, x)$ is a weak solution of equation (5.1), i.e.

$$
\partial_{t} u=\delta \Delta u+\nabla \cdot \Psi(u) \quad \text { with } \Psi(u)=|u| \nabla^{\alpha-1} G(u)
$$

in $(0, T) \times \mathbb{R}^{d}$ in the sense of distributions (cf. equation (5.5)), and

$$
\int u(t, x) \mathrm{d} x=\int u_{0}(x) \mathrm{d} x
$$

for all $t \in(0, T)$.

Proof. If $\alpha \in(0,1]$, for every $u \in \mathcal{C}([0, T], X)$, where the space $X$ is defined in (5.10), we obviously have $u \in L^{\infty}\left((0, T), L^{\infty}\left(\mathbb{R}^{d}\right)\right)$. We derive from inequality 3.6 that for all $p \in\left(\bar{p}_{\alpha}, \infty\right)$, we have $\nabla^{\alpha-1} G(u) \in$ $L^{\infty}\left((0, T), L^{p}\left(\mathbb{R}^{d}\right)\right)$ with $\bar{p}_{\alpha}=\frac{d}{d-(1-\alpha)}>1$. This implies

$$
\nabla \cdot \Psi(u) \in L^{q}\left((0, T), H^{-1, p}\left(\mathbb{R}^{d}\right)\right)
$$

for all $q \in(1, \infty)$ and $p \in\left(\bar{p}_{\alpha}, \infty\right)$. Thus, the maximal regularity of mild solutions for the nonhomogeneous heat equation [17] gives us $\nabla u \in L^{q}\left((0, T), L^{p}\left(\mathbb{R}^{d}\right)\right)$ for every $q \in(1, \infty)$ and $p \in\left(\bar{p}_{\alpha}, \infty\right)$. Consequently, $\nabla \cdot\left(|u| \nabla^{\alpha-1} G(u)\right)=f_{1}+f_{2}$ with

$$
f_{1}=\nabla|u| \cdot \nabla^{\alpha-1} G(u), \quad f_{2}=-|u|(-\Delta)^{\frac{\alpha}{2}} G(u)
$$

First, we remark that $f_{1} \in L^{q}\left((0, T), L^{p}\left(\mathbb{R}^{d}\right)\right)$ for every $q \in(1, \infty)$ and $p \in\left(\bar{p}_{\alpha}, \infty\right)$. Second, we notice that $G(u) \in L^{q}\left((0, T), H^{1, p}\left(\mathbb{R}^{d}\right)\right)$, hence $(-\Delta)^{\frac{\alpha}{2}} G(u) \in L^{q}\left((0, T), H^{1-\alpha, p}\left(\mathbb{R}^{d}\right)\right) \subset L^{q}\left((0, T), L^{p}\left(\mathbb{R}^{d}\right)\right)$. Hence, we also have $f_{2} \in L^{q}\left((0, T), L^{p}\left(\mathbb{R}^{d}\right)\right)$ for every $q \in(1, \infty)$ and $p \in\left(\bar{p}_{\alpha}, \infty\right)$. Using again the maximal regularity result, we obtain that

$$
\partial_{t} u \in L^{q}\left((0, T), L^{p}\left(\mathbb{R}^{d}\right)\right)
$$

for every $q \in(1, \infty)$ and $p \in\left(\bar{p}_{\alpha}, \infty\right)$. Thus, using the following representation in $L^{p}\left(\mathbb{R}^{d}\right)$ for all $0<s<t$

$$
u(t)-u(s)=\int_{s}^{t} \partial_{t} u(\tau) \mathrm{d} \tau
$$

and the Hölder inequality we obtain for every $p \in\left(\bar{p}_{\alpha}, \infty\right)$

$$
\|u(t)-u(s)\|_{p} \leq \int_{s}^{t}\left\|\partial_{t} u(\tau)\right\|_{p} \mathrm{~d} \tau \leq\left(\int_{s}^{t}\left\|\partial_{t} u(\tau)\right\|_{p}^{q} \mathrm{~d} \tau\right)^{\frac{1}{q}}(t-s)^{\frac{1}{q^{*}}}
$$


i.e. $u \in \mathcal{C}^{0, \beta}\left((0, T), L^{p}\left(\mathbb{R}^{d}\right)\right)$ for all $p \in\left(\bar{p}_{\alpha}, \infty\right)$ and $\beta \in(0,1)$. Using inequality (5.15), this estimate implies that $\Psi(u) \in \mathcal{C}^{0, \beta}\left((0, T), L^{p}\left(\mathbb{R}^{d}\right)\right)$ for every $p \in\left(\bar{p}_{\alpha}, \infty\right)$. Now, the classical theory of linear parabolic equations, see, e.g., [20, Ch. 4, Theorem 3.5], implies that $\partial_{t} u \in \mathcal{C}^{0, \beta}\left((0, T), L^{p}\left(\mathbb{R}^{d}\right)\right)$ and $u \in \mathcal{C}^{0, \beta}\left((0, T), H^{1, p}\left(\mathbb{R}^{d}\right)\right)$ which is the desired regularity result.

If $\alpha \in(1,2)$, we have $u \in L^{q}\left((0, T), H^{\alpha-1, p}\left(\mathbb{R}^{d}\right)\right)$ for all $q \in(1, \infty)$ and $p \in\left(p_{\alpha}, \infty\right)$, where $p_{\alpha}=\bar{p}_{\alpha}=$ $d /(\alpha-1)$. Equations (3.5) and (5.20) imply that

$$
\nabla^{\alpha-1} G(u) \in L^{q}\left((0, T), L^{p}\left(\mathbb{R}^{d}\right)\right)
$$

for all $p \in\left(p_{\alpha}, \infty\right)$ and $q \in(1, \infty)$. In particular, $\Psi(u)=|u| \nabla^{\alpha-1} G(u) \in L^{q}\left((0, T), L^{p}\left(\mathbb{R}^{d}\right)\right)$. Hence, the maximal regularity gives us

$$
u \in L^{q}\left((0, T), H^{1, p}\left(\mathbb{R}^{d}\right)\right)
$$

for all $p \in\left(p_{\alpha}, \infty\right)$ and $q \in(1, \infty)$. We now write once again $\nabla \cdot \Psi(u)=f_{1}+f_{2}$ with

$$
f_{1}=\operatorname{sgn} u \nabla u \cdot \nabla^{\alpha-1} G(u), \quad f_{2}=|u| \nabla^{\alpha-1}\left(G^{\prime}(u) \nabla u\right) .
$$

In view of (5.31) and (5.32), we have

$$
f_{1} \in L^{q}\left((0, T), L^{p}\left(\mathbb{R}^{d}\right)\right) .
$$

We now claim that

$$
f_{2} \in L^{q}\left((0, T), H^{-1+(2-\alpha), p}\left(\mathbb{R}^{d}\right)\right) .
$$

Indeed,

$$
G^{\prime}(u) \nabla u \in L^{q}\left((0, T), L^{p}\left(\mathbb{R}^{d}\right)\right) .
$$

This implies that

$$
\nabla^{\alpha-1}\left(G^{\prime}(u) \nabla u\right) \in L^{q}\left((0, T), H^{-1+(2-\alpha), p}\left(\mathbb{R}^{d}\right)\right)
$$

which, in turn, implies the claim. Hence,

$$
\nabla \cdot \Psi(u) \in L^{q}\left((0, T), H^{-1+(2-\alpha), p}\left(\mathbb{R}^{d}\right)\right)
$$

for all $p \in\left(p_{\alpha}, \infty\right)$ and $q \in(1, \infty)$. Then, the maximal regularity implies that

$$
u \in L^{q}\left((0, T), H^{1+(2-\alpha), p}\left(\mathbb{R}^{d}\right)\right)
$$

for all for all $p \in\left(p_{\alpha}, \infty\right)$ and $q \in(1, \infty)$, thus we see that the space regularity of $u$ is improved. More generally, the same argument shows that if

$$
u \in L^{q}\left((0, T), H^{\beta, p}\left(\mathbb{R}^{d}\right)\right), \quad \text { with } \beta \leq \alpha,
$$

then

$$
u \in L^{q}\left((0, T), H^{\beta+(2-\alpha), p}\left(\mathbb{R}^{d}\right)\right) .
$$

Now choose the least integer $k \geq 1$ such that $\beta_{k}=1+k(2-\alpha)>\alpha$, and notice that $\beta_{k}<2$. Then

$$
f_{2} \in L^{q}\left((0, T), H^{\beta_{k}-\alpha, p}\left(\mathbb{R}^{d}\right)\right) \subset L^{q}\left((0, T), L^{p}\left(\mathbb{R}^{d}\right)\right) .
$$


Then the maximal regularity implies that $\partial_{t} u \in L^{q}\left((0, T), L^{p}\left(\mathbb{R}^{d}\right)\right)$, which implies, as was in the case $\alpha \in(0,1]$, that $u \in \mathcal{C}^{0, \beta}\left((0, T), L^{p}\left(\mathbb{R}^{d}\right)\right)$ for all $p \in(1, \infty)$ and $\beta \in(0,1)$. The previous reasoning in spaces of the form $L^{q}((0, T), Y)$ extends readily to spaces of the form $\mathcal{C}^{0, \beta}((0, T), Y)$. This yields the desired regularity result in the case $\alpha \in(1,2)$.

Moreover, it is known by [20, Ch. 4, Theorem 3.2] that mild solutions of the equation $\partial_{t} u=\delta \Delta u+\nabla \cdot f$ with $f \in \mathcal{C}^{0, \beta}\left((0, T), L^{p}\left(\mathbb{R}^{d}\right)\right)$ are in fact weak solutions, i.e. they satisfy the equation in the sense of distributions. Under these regularity properties, the proof of the mass conservation property (5.29) is completely standard.

Convexity inequalities. First, we show a simple but useful technical result involving monotone functions and the fractional Laplacian.

Lemma 5.8. Let $\alpha \in(0,2]$. Assume that $g, h \in \mathcal{C}^{1}[0, \infty)$ are strictly increasing functions. Then, for every nonnegative $v \in \mathcal{C}_{c}^{\infty}\left(\mathbb{R}^{d}\right)$ we have

$$
\int h(v)(-\Delta)^{\frac{\alpha}{2}} g(v) \mathrm{d} x \geq 0
$$

Proof. Notice that for $\alpha=2$ this lemma is obviously true, which one checks integrating by parts. Now, let $\alpha \in(0,2)$. Since $(-\Delta)^{\frac{\alpha}{2}} C=0$ for every constant $C \in \mathbb{R}$, we can assume that $g(0)=0$. In the same way, we can assume that $h(0)=0$, because $\int(-\Delta)^{\frac{\alpha}{2}} w \mathrm{~d} x=0$ for every $w \in \mathcal{C}_{c}^{\infty}\left(\mathbb{R}^{d}\right)$. Defining $w=g(v)$, it suffices to show that

$$
\int h\left(g^{-1}(w)\right)(-\Delta)^{\frac{\alpha}{2}} w \mathrm{~d} x \geq 0
$$

for all $w \in \mathcal{C}_{c}^{\infty}\left(\mathbb{R}^{d}\right)$ such that $w \geq 0$. To do it, notice that $f \in \mathcal{C}^{2}[0, \infty)$ defined via the relation $f(s)=$ $\int_{0}^{s} h\left(g^{-1}(\tau)\right) \mathrm{d} \tau$ for $s \geq 0$ is convex (it suffices to check that $f^{\prime \prime}(s) \geq 0$ ). Hence, using the pointwise inequality

$$
(-\Delta)^{\frac{\alpha}{2}} g(v) \leq g^{\prime}(v)(-\Delta)^{\frac{\alpha}{2}} v
$$

(see, e.g., 9], 12, Lemma 1]) we obtain $\int h\left(g^{-1}(w)\right)(-\Delta)^{\frac{\alpha}{2}} w \mathrm{~d} x \geq \int(-\Delta)^{\frac{\alpha}{2}} f(w) \mathrm{d} x=0$.

Next, we formulate a crucial technical tool used in the derivation of various integral estimates for solutions of the regularized problem (5.1).

Proposition 5.9 (Convexity inequalities). Consider a $\mathcal{C}^{2}$ function $\varphi: \mathbb{R} \rightarrow \mathbb{R}^{+}$such that, for all $r \in \mathbb{R}, r \neq 0$, $\varphi^{\prime \prime}(r)>0$, and

$$
\varphi(r)+\left|\varphi^{\prime}(r)\right|+\varphi^{\prime \prime}(r) \leq C\left(|r|^{M_{1}}+|r|^{M_{2}}\right)
$$

for some constant $C>0$ and $M_{1}, M_{2} \in[1, \infty)$. Then for all $0 \leq s<t \leq T$, the function $u$ given by Proposition 5.2 satisfies

$$
\int \varphi(u(t, x)) \mathrm{d} x+\int_{s}^{t} \int \psi(u(\tau, x))(-\Delta)^{\frac{\alpha}{2}} G(u(\tau, x)) \mathrm{d} x \mathrm{~d} \tau+\delta \int_{s}^{t} \int \varphi^{\prime \prime}(u)|\nabla u|^{2} \mathrm{~d} x \mathrm{~d} \tau \leq \int \varphi(u(s, x)) \mathrm{d} x,
$$
where $\psi(r)=|r| \varphi^{\prime}(r)-\varphi(r) \operatorname{sgn} r$. 
Remark 5.10. Remark that $\psi^{\prime}(r)=|r| \varphi^{\prime \prime}(r)>0$, hence the function $\psi$ is increasing. Since $G$ is also increasing, the result stated in Lemma 5.8 can be applied to show that the first dissipation term

$$
\int_{s}^{t} \int \psi(u(\tau, x))(-\Delta)^{\frac{\alpha}{2}} G(u(\tau, x)) \mathrm{d} x \mathrm{~d} \tau
$$

is nonnegative. The fact that this quantity is finite is a part of the result stated in Proposition 5.9 , Moreover, [18. Theorem 2.2] implies that $g_{\varphi}(G(u)) \in L^{2}\left((0, T), H^{\frac{\alpha}{2}, 2}\left(\mathbb{R}^{d}\right)\right)$ for a function $g_{\varphi}$ constructed from $\varphi$, see [18] for the detailed presentation. The special case $\varphi(r)=|r|^{p}$ is treated below.

Remark 5.11. The convexity of $\varphi$ also implies that the second dissipative term in (5.35) is nonnegative. Hence, Proposition 5.9 implies that $\int \varphi(u(t, x)) \mathrm{d} x$ decreases along the flow of the regularized equation (5.1).

Corollary 5.12 (Estimates of the $L^{p}$-norms). For all $p \in(1, \infty)$ and $0<s<t$,

$$
\int|u(t)|^{p} \mathrm{~d} x+(p-1) \int_{s}^{t} \int|u|^{p-1} u(-\Delta)^{\frac{\alpha}{2}} G(u) \mathrm{d} x \mathrm{~d} \tau+\delta p(p-1) \int_{s}^{t} \int|u|^{p-2}|\nabla u|^{2} \mathrm{~d} \tau \leq \int|u(s)|^{p} \mathrm{~d} x
$$

In particular, for $p \geq \bar{p}_{\alpha}$ (see Corollary 5.7),

$$
\frac{\mathrm{d}}{\mathrm{d} t} \int|u|^{p} \mathrm{~d} x \leq-(p-1) \int|u|^{p-1} u(-\Delta)^{\frac{\alpha}{2}} G(u) \mathrm{d} x-\delta p(p-1) \int|u|^{p-2}|\nabla u|^{2} .
$$

Thus, for all $p \in[1, \infty]$, the norm $\|u(t)\|_{p}$ decreases as $t$ increases.

Proof. If $p \geq 3$, we can apply Proposition 5.9 with $0 \leq s<t \leq T, \varphi(r)=|r|^{p}$; indeed, in this case, $\varphi$ is a $\mathcal{C}^{2}$-function and satisfies the growth assumption with with $\left\langle M_{1}, M_{2}\right\rangle=\langle p, p-2\rangle$. Next, since $u \in$ $\mathcal{C}^{1}\left((0, T), L^{p}\left(\mathbb{R}^{d}\right)\right)$, we obtain (5.37) from the inequality in Proposition 5.9 by a direct computation. We leave the details to the reader.

If $p \in(1,2)$, we consider, for each $\eta>0$, the function $\varphi_{\eta}$ such that $\varphi_{\eta}(0)=\varphi_{\eta}^{\prime}(0)=0$ and

$$
\varphi_{\eta}^{\prime \prime}(r)=p(p-1)\left(\left(r^{2}+\eta^{2}\right)^{\frac{p}{2}-1}-\eta^{p-2}\right)
$$

In particular, the function $\varphi_{\eta}$ satisfies the assumptions of Proposition 5.9, hence, we have

$$
\begin{aligned}
\int \varphi_{\eta}(u(t, x)) \mathrm{d} x+\int_{s}^{t} \int \psi_{\eta}(u(s, x))(-\Delta)^{\frac{\alpha}{2}} G(u(s, x)) \mathrm{d} x \mathrm{~d} s & \\
& +\delta \int_{s}^{t} \int \varphi_{\eta}^{\prime \prime}(u(s, x))|\nabla u(s, x)|^{2} \mathrm{~d} x \mathrm{~d} s \leq \int \varphi_{\eta}(u(s, x)) \mathrm{d} x,
\end{aligned}
$$

with $\psi_{\eta}^{\prime}(r)=|r| \varphi_{\eta}^{\prime \prime}(r), \psi_{\eta}(0)=0$. Letting now $\eta \rightarrow 0$ and using the Fatou lemma yields the integral formulation of inequality (5.37).

Thus, we just proved that $\|u(t)\|_{p} \leq\left\|u_{0}\right\|_{p}$ for all $t>0$ and $p \in(1, \infty)$. By computing the limits as $p \rightarrow \infty$ and $p \rightarrow 1$, the bounds $\|u(t)\|_{1} \leq\left\|u_{0}\right\|_{1}$ and $\|u(t)\|_{\infty} \leq\left\|u_{0}\right\|_{\infty}$ are also obtained.

We can now complete the proof of Theorem 5.1 .

Proof of Theorem 5.1. In view of Proposition 5.2 and Corollary 5.7 it remains to prove that solutions are nonnegative if initial data are so, and that solutions are global in time. 
The positivity property is derived immediately in a usual way from the conservation of mass property (5.6) and the monotonicity of the $L^{1}$-norm. Indeed, (5.6) yields

$$
\int u(T, x) \mathrm{d} x=\int u_{+}(T, x) \mathrm{d} x-\int u_{-}(T, x) \mathrm{d} x=\int\left(\left(u_{0}\right)_{+}-\left(u_{0}\right)_{-}\right)(x) \mathrm{d} x
$$

and

$$
\int|u(T, x)| \mathrm{d} x=\int u_{+}(T, x) \mathrm{d} x+\int u_{-}(T, x) \mathrm{d} x \leq \int\left(\left(u_{0}\right)_{+}+\left(u_{0}\right)_{-}\right)(x) \mathrm{d} x
$$

(here, as usual, $u_{+}=\max \{0, u\}$ and $\left.u_{-}=\max \{0,-u\}\right)$. These inequalities imply $\int u_{-}(T, x) \mathrm{d} x \leq \int\left(u_{0}\right){ }_{-}(x) \mathrm{d} x$, and, in particular, the assumption $\left(u_{0}\right)_{-}=0$ gives us $u \geq 0$ a.e.

As far as the global existence of solutions is concerned, we argue as follows.

For $\alpha \in(0,1]$, the time interval, where a solution is constructed via the Banach fixed point theorem, depends only on $\left\|u_{0}\right\|_{1}+\left\|u_{0}\right\|_{\infty}$, and this norm of the solution does not increase. Hence, we extend $u=u(t, \cdot)$ to the whole half-line $[0, \infty)$, step-by-step.

For $\alpha \in(1,2)$, the Duhamel formula (5.8) and inequality (5.20) with $v=0$ yield

$$
\|u(t)\|_{H^{\alpha-1, p}} \leq\left\|u_{0}\right\|_{H^{\alpha-1, p}}+C_{1}\left(\left\|u_{0}\right\|_{p^{*}}\right) \int_{0}^{t}(t-s)^{-\frac{\alpha}{2}}\|u(s)\|_{H^{\alpha-1, p}} \mathrm{~d} s .
$$

Due to the singular Gronwall lemma, see, e.g., [20, Ch. 5, Lemma 6.7], we deduce that the norm $\|u(t)\|_{H^{\alpha-1, p}}$ cannot explode in finite time. This shows that local-in-time solutions of the regularized equation (5.1) can be also continued to global-in-time ones.

\section{HyPERCONTRACTIVITY AND COMPACTNESS ESTIMATES}

Hypercontractivity estimates. We now turn to prove certain $L^{1} \mapsto L^{p}$ estimates for solutions of problem (5.1).

Theorem 6.1 ( $L^{p}$-decay of solutions to the regularized problem). Let $u=u(t, x)$ be a solution to the regularized problem (5.1) constructed in Theorem 5.1. There exists a constant $C=C(d, \alpha, m)>0$ such that for all $\varepsilon>0$, $\delta>0$ and $p \in[1, \infty]$,

$$
\|u(t)\|_{p} \leq C\left\|u_{0}\right\|_{1}^{\frac{d(m-1) / p+\alpha}{d(m-1)+\alpha}} t^{-\frac{d}{d(m-1)+\alpha}}\left(1-\frac{1}{p}\right)
$$

for all $t>0$.

Proof. We first remark that it is enough to prove the decay estimate (6.1) for large $p$ 's, since the general result follows by the interpolation of the $L^{p}$-norms combined with the estimate $\|u(t)\|_{1} \leq\left\|u_{0}\right\|_{1}$ from Corollary 5.12 This is the reason why we will now prove (6.1) for $p \geq \max \left\{m-1,1, \bar{p}_{\alpha}\right\}=p_{m}$ (see Corollary[5.7 for a definition of $\left.\bar{p}_{\alpha}\right)$.

We also remark that we can assume that $M=\left\|u_{0}\right\|_{1}=1$ by rescaling the solution $u$ in the following way. First, we consider the function $\widetilde{u}(t)=\frac{1}{M} u\left(\frac{t}{M^{m-1}}\right)$ which satisfies equation (5.1) with suitably rescaled parameters: $\tilde{\delta}=\frac{\delta}{M^{m-1}}$ and (if applicable) $\tilde{\varepsilon}=\frac{\varepsilon}{M}$. Scaling back, we recover the desired inequality (6.1). 
We first prove the result when Corollary 5.12 holds true with $G(r)=|r|^{m-2} r$ and in the differential form (5.37). This is the case when $m=2$ or $m \geq 3$. In the case $m \in(1,3)$, Corollary 5.12 holds true only in the integral sense (5.36) and for a regularized function $G$. We will see below how to pass to the limit as the regularization parameter $\varepsilon$ goes to 0 and get (5.36) with $G(r)=|r|^{m-2} r$. For expository reasons, we prefer to present the proof when we indeed have a differential inequality, and then to explain how to adapt it if only an integral version of it is available.

The proof on Theorem 6.1 in the cases $m \geq 3$ and $m=2$ is split into two steps: first, we show inequalities (6.1) with non-optimal constants $C$ which blow up for $p=\infty$; then, we improve those constants by an iteration method.

Decay estimates with optimal exponents and nonoptimal constants. Our computation consists in getting the following differential inequality for $p \in\left(p_{m}, \infty\right)$.

Lemma 6.2. There exists a constant $K=K(p, m)>0$ independent of $\varepsilon>0$ and $\delta>0$ such that $K$ and $K^{-1}$ are bounded as $p \rightarrow \infty$ and

$$
\frac{\mathrm{d}}{\mathrm{d} t}\|u\|_{p}^{p} \leq-K\|u\|_{p}^{a}
$$

with a defined in (3.9).

Proof. We get from Corollary 5.12

$$
\begin{aligned}
\frac{\mathrm{d}}{\mathrm{d} t} \int|u|^{p} \mathrm{~d} x & \leq-(p-1) \int u|u|^{p-1}(-\Delta)^{\frac{\alpha}{2}}\left(u|u|^{m-2}\right) \mathrm{d} x \\
& \leq-\frac{4 p(p-1)(m-1)}{(p+m-1)^{2}}\left\|\nabla^{\frac{\alpha}{2}}\left(|u|^{\frac{p+m-1}{2}}\right)\right\|_{2}^{2},
\end{aligned}
$$

after applying the Stroock-Varopoulos inequality (3.4) with $w=u|u|^{m-2}$ and $q=\frac{p}{m-1}+1$. We use next the Gagliardo-Nirenberg inequality from Lemma 3.2 combined with $\|u(t)\|_{1} \leq\left\|u_{0}\right\|_{1}=1$ to estimate the right-hand side of the above inequality. Thus, we get the differential inequality (6.2) with the constant

$$
K=\frac{4(m-1) p(p-1)}{C_{N}(p+m-1)^{2}}
$$

With the differential inequality (6.2) in hand, a direct computation shows that every nonnegative solution of the inequality $\frac{\mathrm{d}}{\mathrm{d} t} f(t) \leq-K f(t)^{\frac{a}{p}}$ has to satisfy the algebraic decay

$$
f(t) \leq\left(K\left(\frac{a}{p}-1\right) t\right)^{-\frac{a}{\frac{a}{p}-1}} .
$$

We recognize (6.1) for $p>p_{m}$ with the constant $C_{p}=\left(K\left(\frac{a}{p}-1\right)\right)^{-\frac{1}{a-p}}$. Here, let us notice that the constants $C_{p}=C(d, \alpha, m, p)$ which are obtained at this stage of the proof blow up as $p \rightarrow \infty$. Thus, we cannot get the $L^{\infty}$-bound directly in this way. 
Recurrence step. To improve the constant $C_{p}$ and to handle the limit case $p=\infty$, we apply a variation on the Moser-Alikakos method of estimating the $L^{p}$-norms with $p=2^{n}$ recursively, see, e.g., [1] and [16, Lemma 3.1]. The starting point is the already obtained estimate for $p=2^{k} \geq m-1$ with the least integer $k$.

Lemma 6.3. For each $n \geq k$, the following estimate

$$
\|u(t)\|_{2^{n}} \leq \kappa_{n} t^{-\mu_{n}} \quad \text { for all } t>0
$$

holds true with $\mu_{n}=\frac{1-2^{-n}}{\frac{\alpha}{d}+m-1}$ and with a positive $\kappa_{n}$ satisfying the recursive estimate

$$
\kappa_{n+1} \leq\left[\frac{2^{n}\left(\frac{2 \alpha}{d}+\frac{m-1}{2^{n}}\right) \mu_{n}+1}{K_{n}\left(\frac{\alpha}{d}+\frac{m-1}{2^{n}}\right)} \kappa_{n}^{2^{n}\left(\frac{2 \alpha}{d}+\frac{m-1}{2^{n}}\right)}\right]^{2^{-n-1} \frac{1}{\frac{\alpha}{d}+\frac{m-1}{2^{n}}}},
$$

where $K_{n}$ is given by formula (6.4) with $p=2^{n+1}$.

Thus, having this estimate we see that $\lim _{\sup _{n \rightarrow \infty}} \kappa_{n}<\infty$ (irrespective of the value of $\kappa_{k}$ at the beginning of the recurrence), essentially since $\sum_{n=k}^{\infty} n 2^{-n}<\infty$, see Appendix B for details. Recall that the constants $K=K_{n}$ in the preliminary estimates have been such that $K_{n}$ and $K_{n}^{-1}$ were bounded uniformly when $p=$ $2^{n} \rightarrow \infty$.

Proof of Lemma 6.3. We combine (6.3) with the Nash inequality (3.10) and two Hölder inequalities with $2^{n+1}<$ $r=2^{n+1}+m-1 \leq 2^{n+2}$

$$
\|u\|_{2^{n+1}} \leq\|u\|_{r}^{\gamma}\|u\|_{2^{n}}^{1-\gamma} \quad \text { with } \quad \frac{1}{\gamma}=2-\frac{2^{n+1}}{r}
$$

and

$$
\|u\|_{\frac{r}{2}} \leq\|u\|_{2^{n+1}}^{\delta}\|u\|_{2^{n}}^{1-\delta} \quad \text { with } \quad \delta=2-\frac{2^{n+2}}{r}
$$

As the result, we have

$$
\frac{\mathrm{d}}{\mathrm{d} t}\|u\|_{2^{n+1}}^{2^{n+1}} \leq-K\|u\|_{2^{n+1}}^{2^{n+1}\left(1+\frac{\alpha}{d}+\frac{m-1}{2^{n}}\right)}\|u\|_{2^{n}}^{-2^{n}}\left(\frac{2 \alpha}{d}+\frac{m-1}{2^{n}}\right)
$$

with some $K$ as in (6.2) corresponding to $p=2^{n+1}$. Next we estimate the $L^{2^{n}}$-norm of $u(t)$ using (6.5), and arrive to the differential inequality for $f(t)=\|u(t)\|_{2^{n+1}}^{2^{n+1}}$ of the form

$$
\frac{\mathrm{d}}{\mathrm{d} t}\left(f(t)^{-\frac{\alpha}{d}-\frac{m-1}{2^{n}}}\right) \geq \frac{K\left(\frac{\alpha}{d}+\frac{m-1}{2^{n}}\right)}{\kappa_{n}^{2^{n}\left(\frac{2 \alpha}{d}+\frac{m-1}{2^{n}}\right)}} t^{\mu_{n} 2^{n}\left(\frac{2 \alpha}{d}+\frac{m-1}{2^{n}}\right)} .
$$

Finally, we integrate this inequality on $[0, t]$ and take a suitable negative power to arrive to (6.6).

Interpolation between $p=2^{n}$ and $p=2^{n+1}$ and the passage to the limit $p \rightarrow \infty$ finish the proof of the hypercontractivity estimates in the cases $m \geq 3$ and $m=2$.

To deal with the case $m \in(1,3)$, instead of the differential inequality from Corollary 5.12, we use its integral counterpart (5.36). In particular, inequalities (6.2) and (6.7) have their integral counterparts as well (see inequality (7.5), below). Hence, the previous proof works in this case by applying the following lemma with $g(t)=t$ and $g(t)=C t^{\nu}$ for some well chosen positive constants $\nu, C$, successively. 
Lemma 6.4. Consider functions $f:[0, T] \rightarrow(0, \infty)$ nonincreasing, and $g:[0, T] \rightarrow(0, \infty)$ increasing, smooth, and $g(0)=0$. Assume that for a.e. $t \in(0, T), s<t$,

$$
f(t)+K \int_{s}^{t} f(\tau)^{\gamma+1} g^{\prime}(\tau) \mathrm{d} \tau \leq f(s) .
$$

Then for a.e. $t \in[0, T]$, we have

$$
f(t) \leq(K \gamma g(t))^{-\frac{1}{\gamma}}
$$

The proof of this lemma is given in Appendix $\mathrm{C}$ for the readers' convenience. Now, the proof of Theorem 6.1 is complete.

Compactness estimates. Now, we prove estimates which will allow us to pass to the limit as $\varepsilon \rightarrow 0$ and $\delta \rightarrow 0$, successively, in the regularized problem (5.1). We are going to use the fact that the approximating functions $G=G_{\varepsilon}$ are $\gamma$-Hölder continuous with $\gamma=\min \{m-1,1\}$ on the interval $\left[0,\left\|u_{0}\right\|_{\infty}\right]$, uniformly in $\varepsilon \in[0,1]$. These estimates are formulated in the following technical lemmas.

Lemma 6.5. Assume that $G$ is $\gamma$-Hölder continuous with $\gamma=\min \{m-1,1\}$. For every $\alpha \in(0,1]$ and $m>1+\frac{1-\alpha}{d} \in(1,2)$, we have

$$
\left\|\nabla^{\alpha-1} G(u)\right\|_{q} \leq C\|u\|_{\gamma p}^{\gamma}
$$

with

$$
q \geq(m-1-(1-\alpha) / d)^{-1} \text { and } p=(1 / q+(1-\alpha) / d)^{-1}
$$

Proof. Here, it suffices to combine the estimate on the Riesz potential with $\|G(u)\|_{p} \leq C\|u\|_{\gamma p}^{\gamma}$. The choice of $q$ (or equivalently, the restriction on $m$ ) ensures that $\gamma p \geq 1$.

The compactness of a sequence of solutions to the regularized problem (5.1) in the case $\alpha \in(1,2)$ is a consequence of the following estimate.

Lemma 6.6. Consider a $\gamma$-Hölder continuous function $G: \mathbb{R} \rightarrow \mathbb{R}$. Then, for every $\alpha \in(1,2)$ such that $(2-\gamma) \alpha<2$, we have

$$
\left\|\nabla^{\alpha-1} G(u)\right\|_{p} \leq C\|u\|_{H^{\frac{\alpha}{2}, 2}}
$$

with

$$
\frac{\alpha-1}{\gamma}-\frac{d}{p}=\frac{\alpha}{2}-\frac{d}{2}
$$

Remark 6.7. Note that the assumptions of Lemma 6.6 ensure that $p>2$.

Proof of Lemma 6.6. We use successively the fact that $H^{s, p}\left(\mathbb{R}^{d}\right)=F_{p, 2}^{s}\left(\mathbb{R}^{d}\right)$ [22, p.14], the characterization of Triebel-Lizorkin spaces with difference quotients [22, p.41], and finally known embedding theorems for Besov and Triebel-Lizorkin spaces [22, p.31] in order to derive

$$
\left\|\nabla^{\alpha-1} G(u)\right\|_{p} \leq C\|G(u)\|_{F_{p, 2}^{\alpha-1}} \leq C[G]_{\gamma}\|u\|_{F_{p, 2 \gamma}^{\frac{\alpha-1}{\gamma}}} \leq C\|u\|_{H^{\frac{\alpha}{2}, 2}}
$$


where $[G]_{\gamma}:=\sup _{r \neq s}|G(r)-G(s)| /|r-s|^{\gamma}$ and $p>2$ is chosen so that the so-called differential dimension is constant; this yields the condition appearing in the statement of the lemma. The proof is now complete.

Lemma 6.8. For $\alpha \in(1,2)$ and $(3-m) \alpha<2$, there exists $p \in(1, \infty)$ such that

$$
\left\|\nabla^{\alpha-1} G(u)\right\|_{p} \leq C\|u\|_{H^{1,2}\left(\mathbb{R}^{d}\right)}
$$

where $C$ depends on the $\gamma$-Hölder seminorm of $G$ in $\left(0,\|u\|_{\infty}\right)($ with $\gamma=\min \{m-1,1\})$.

Proof. Apply Lemma 6.6 with $\gamma=m-1$.

Lemma 6.9. If $\alpha \in(1,2)$ and $m>\alpha$, there exists $p \in(1, \infty)$ and $r \in(1, \infty)($ and $r>m-1)$ such that

$$
\left\|\nabla^{\alpha-1}|u|^{m-1} \operatorname{sgn} u\right\|_{p} \leq C\left\||u|^{\frac{r+m-1}{2}} \operatorname{sgn} u\right\|_{H^{\frac{\alpha}{2}, 2}\left(\mathbb{R}^{d}\right)} .
$$

Proof. Consider $v=|u|^{\frac{r+m-1}{2}} \operatorname{sgn} u$. Then estimate (6.10) is equivalent to the following one

$$
\left\|\nabla^{\alpha-1}|v|^{\frac{2(m-1)}{r+m-1}} \operatorname{sgn} v\right\|_{p} \leq C\|v\|_{H^{\frac{\alpha}{2}, 2}\left(\mathbb{R}^{d}\right)} .
$$

We then apply Lemma 6.6 with $\gamma=\frac{2(m-1)}{r+m-1}$ and get the desired result if $\gamma>2\left(1-\frac{1}{\alpha}\right)$, or equivalently,

$$
r<\frac{m-1}{\alpha-1}
$$

Here, in order to find $r \in\left(1, \frac{m-1}{\alpha-1}\right)$, the condition $m>\alpha$ is needed. The proof is now complete.

\section{Proof of Theorem 2.6}

This section is devoted to the proof of our main result on the existence of solutions to problem (1.1)-(1.2) satisfying decay estimates (2.6).

Proof of Theorem 2.6. We first consider very regular initial data, i.e. we assume that $u_{0}$ satisfies (5.3). Then, this condition is relaxed by considering initial data that are merely integrable.

The proof proceeds in three steps: passage to the limit with the parameter of the regularization of the nonlinearity, then with the parameter of the parabolic regularization, and finally — stability with respect to initial data.

Passage to the limit as $\varepsilon \rightarrow 0$. Consider $u_{0}$ satisfying (5.3). From Theorem [5.1, we have a sequence of solutions $u_{\varepsilon}$ of (5.1) for $G_{\varepsilon}$ defined in such a way that there exists $C>0$ such that for all $\varepsilon \in(0,1]$,

$$
\left[G_{\varepsilon}\right]_{\gamma}:=\sup _{r \neq s} \frac{\left|G_{\varepsilon}(r)-G_{\varepsilon}(s)\right|}{|r-s|^{\gamma}} \leq C
$$

where $\gamma=\min \{m-1,1\}$. Thanks to Corollary [5.12, there exists also a constant $C>0($ depending on $\delta>0)$ such that for all $\varepsilon \in(0,1]$,

$$
\left\|u_{\varepsilon}\right\|_{L^{\infty}\left((0, T) \times \mathbb{R}^{d}\right)} \leq C, \quad\left\|u_{\varepsilon}\right\|_{L^{2}\left((0, T), H^{1,2}\left(\mathbb{R}^{d}\right)\right)} \leq C .
$$

Hence, we can construct a sequence $\left(\varepsilon_{n}\right)_{n}$ such that

$$
u_{n} \rightarrow u \text { in } L^{2}\left((0, T), H^{1,2}\left(\mathbb{R}^{d}\right)\right)
$$


where $u_{n}$ denotes $u_{\varepsilon_{n}}$. Moreover, for all $R>0$, the embedding $H^{1,2}\left(B_{R}\right) \subset L^{2}\left(B_{R}\right)$ is dense and compact. Using (7.1) we also obtain

$$
\lim _{\operatorname{meas}(E) \rightarrow 0, E \subset[0, T]} \int_{E} \int_{B_{R}}\left|u_{n}(t, x)\right|^{2} \mathrm{~d} t \mathrm{~d} x=0 .
$$

Hence, we infer from 21] (which contains an optimal result on the compactness for Hilbert space valued vector functions) that, up to a subsequence, for every $R>0$,

$$
u_{n} \rightarrow u \text { in } L^{2}\left((0, T) \times B_{R}\right) .
$$

Moreover, passing again to a subsequence if necessary, we can assume that for every $R>0$,

$$
\nabla u_{n} \rightarrow \nabla u \text { in } L^{2}\left((0, T) \times B_{R}\right), \quad u_{n} \rightarrow u \text { for a.e. }(t, x) \in Q_{T},
$$

which imply for all $\varphi \in \mathcal{C}_{c}^{\infty}\left(\bar{Q}_{T}\right)$,

$$
\begin{gathered}
\iint_{Q_{T}} u_{n} \partial_{t} \varphi \mathrm{d} t \mathrm{~d} x \rightarrow \iint_{Q_{T}} u \partial_{t} \varphi \mathrm{d} t \mathrm{~d} x, \\
\iint_{Q_{T}} \nabla u_{n} \cdot \nabla \varphi \mathrm{d} t \mathrm{~d} x \rightarrow \iint_{Q_{T}} \nabla u \cdot \nabla \varphi \mathrm{d} t \mathrm{~d} x .
\end{gathered}
$$

As far as the nonlinear term in equation (5.1) is concerned, we have

$$
\left|u_{n}\right| \rightarrow|u| \text { for a.e. }(t, x) \in Q_{T} \text {. }
$$

Hence, the dominated convergence theorem combined with (7.1) imply that

$$
\left|u_{n}\right| \nabla \varphi \rightarrow|u| \nabla \varphi \text { in } L^{2}\left((0, T), L^{q^{\prime}}\left(\mathbb{R}^{d}\right)\right)
$$

for all $q^{\prime} \in(1, \infty)$. Lemmas 6.5 and 6.8 imply that

$$
\nabla^{\alpha-1} G_{\varepsilon_{n}}\left(u_{n}\right) \text { is bounded in } L^{q}\left(Q_{T}\right)
$$

for some $q \in(1, \infty)$.

We deduce from (17.3) that we can extract a weakly converging subsequence of $\left(\nabla^{\alpha-1} G_{\varepsilon_{n}}\left(u_{n}\right)\right)_{n} \subset L^{q}\left(Q_{T}\right)$ which limit is denoted by $L$. Since

$$
G_{\varepsilon_{n}}\left(u_{n}\right) \rightarrow|u|^{m-1} \operatorname{sgn} u \quad \text { a.e. in } Q_{T},
$$

we have

$$
\nabla^{\alpha-1} G_{\varepsilon_{n}}\left(u_{n}\right) \rightarrow L \text { in } L^{q}\left(Q_{T}\right), \quad \text { where } \quad L=\nabla^{\alpha-1}\left(|u|^{m-1} \operatorname{sgn} u\right) \text { in } \mathcal{D}^{\prime}\left(Q_{T}\right) .
$$

In particular, $\nabla^{\alpha-1}\left(|u|^{m-1} \operatorname{sgn} u\right) \in L^{q}\left(Q_{T}\right)$ holds, and

$$
\iint_{Q_{T}} \nabla^{\alpha-1} G_{\varepsilon_{n}}\left(u_{n}\right) \cdot\left|u_{n}\right| \nabla \varphi \mathrm{d} t \mathrm{~d} x \rightarrow \iint_{Q_{T}} \nabla^{\alpha-1}\left(|u|^{m-1} \operatorname{sgn} u\right) \cdot|u| \nabla \varphi \mathrm{d} t \mathrm{~d} x
$$

as $n \rightarrow \infty$. Hence, we obtain a weak solution of (5.1) with $G(r)=|r|^{m-1} \operatorname{sgn} r$ for $u_{0}$ satisfying (5.3). 
By the Fatou lemma, we derive from Corollary 5.12 and Proposition 3.1 the following estimates: for a.e. $t>s>0, q \in[1, \infty], p \in(1, \infty)$, uniformly in $\delta \in(0,1]$,

$$
\begin{array}{r}
\|u(t)\|_{q} \leq\left\|u_{0}\right\|_{q}, \\
\|u(t)\|_{p}^{p}+\frac{4 p(p-1)(m-1)}{(p+m-1)^{2}} \iint_{(s, t) \times \mathbb{R}^{d}}\left|\nabla^{\frac{\alpha}{2}}\left(\operatorname{sgn} u|u|^{\frac{p+m-1}{2}}\right)\right|^{2} \mathrm{~d} s \mathrm{~d} x \leq\|u(s)\|_{p}^{p}, \\
\|u(t)\|_{q} \leq C\left\|u_{0}\right\|_{1}^{\frac{d(m-1) / q+\alpha}{d(m-1)+\alpha}} t^{-\frac{d}{d(m-1)+\alpha}}\left(1-\frac{1}{q}\right), \\
2 \delta \iint_{Q_{t}}|\nabla u|^{2} \mathrm{~d} s \mathrm{~d} x \leq\left\|u_{0}\right\|_{2}^{2} .
\end{array}
$$

Remark that now we can derive the hypercontractivity estimates in the case $m \in(1,3)$ from inequality (7.5).

We also know that the solution $u$ is nonnegative if $u_{0}$ is so.

Passage to the limit as $\delta \rightarrow 0$. Let $u=u^{\delta}$ denote the solution constructed above. We consider

$$
v^{\delta}=|u|^{m} \operatorname{sgn} u \text {. }
$$

Using (7.5) with $s=0$ and $p=m+1>1$, we get that, for all $T>0$,

$$
v^{\delta} \text { is bounded in } L^{2}\left((0, T), H^{\frac{\alpha}{2}, 2}\left(\mathbb{R}^{d}\right)\right) .
$$

Hence, there exists a subsequence $\delta_{n} \rightarrow 0$ such that

$$
v_{n} \rightarrow v \quad \text { in } L^{2}\left((0, T), H^{\frac{\alpha}{2}, 2}\left(\mathbb{R}^{d}\right)\right),
$$

where $v_{n}$ denotes $v^{\delta_{n}}$. Moreover, inequality (7.4) with $q=\infty$ implies that for $R>0$

$$
\lim _{\operatorname{meas}(E) \rightarrow 0, E \subset[0, T]} \int_{E} \int_{B_{R}}\left|v_{n}(t, x)\right|^{2} \mathrm{~d} t \mathrm{~d} x=0 .
$$

Hence, we can use 21] once more, and conclude that for all $R>0$

$$
v_{n} \rightarrow v \text { in } L^{2}\left((0, T) \times B_{R}\right) .
$$

Moreover, passing to a subsequence if necessary, we can assume that

$$
u_{n} \rightarrow u \text { for a.e. }(t, x) \in Q_{T},
$$

where $u_{n}$ denotes $u^{\delta_{n}}$. Now Lemmas 6.5 and 6.9 imply that

$$
\nabla^{\alpha-1}\left(\left|u_{n}\right|^{m-1} \operatorname{sgn} u_{n}\right) \text { is bounded in } L^{q}\left(Q_{T}\right)
$$

for some $q>1$. Hence, we can pass to the limit in the nonlinear term in the weak formulation of equation (5.1).

To complete this proof, notice that inequality (7.7) implies that

$$
\delta_{n} \nabla u_{n} \rightarrow 0 \text { in } L^{2}\left(Q_{T}\right)
$$

In particular,

$$
\delta_{n} \iint_{Q_{T}} \nabla u_{n} \cdot \nabla \varphi \mathrm{d} t \mathrm{~d} x \rightarrow 0
$$

as $n \rightarrow \infty$. We thus conclude that $u$ is a weak solution of (1.1)-(1.2). 
The conservation of mass, the positivity property, the monotonicity of $L^{p}$-norms, and the hypercontractivity estimates follow from (7.4) - (17.7) in a standard way.

Stability with respect initial conditions. Assume now that $u_{0} \in L^{1}\left(\mathbb{R}^{d}\right) \cap L^{\infty}\left(\mathbb{R}^{d}\right)$. Consider an approximating sequence $u_{0}^{n}$ satisfying (5.3). Then, the sequences of $L^{p}$-norms are bounded and we can pass to the limit as we did already when letting $\delta \rightarrow 0$. We also recover the expected properties of the weak solution.

Assume finally that $u_{0}$ is merely integrable. Then $T_{n}\left(u_{0}\right) \in L^{1}\left(\mathbb{R}^{d}\right) \cap L^{\infty}\left(\mathbb{R}^{d}\right)$ where

$$
T_{n}(r)=\min \{\max \{r,-n\}, n\} .
$$

Hence, there exists a weak solution $u_{n}$ of (1.1). In view of the hypercontractivity estimates, we can extract a converging subsequence in $(\eta, T) \times \mathbb{R}^{d}$, with arbitrary $\eta>0$, to a function $u^{\eta}$ in the following sense

$$
u_{n} \rightarrow u^{\eta} \text { in } L^{2}((\eta, T) \times B(0, R)) .
$$

Moreover, the $L^{1}$-norm of $u_{n}$ is bounded in $(0, T) \times \mathbb{R}^{d}$. Then we have

$$
\begin{aligned}
\left|\iint_{(0, \eta) \times \mathbb{R}^{d}} u_{n} \partial_{t} \varphi \mathrm{d} t \mathrm{~d} x\right| & \leq C \eta, \\
\iint_{(\eta, T) \times \mathbb{R}^{d}} u_{n} \partial_{t} \varphi & \rightarrow \iint_{(\eta, T) \times \mathbb{R}^{d}} u^{\eta} \partial_{t} \varphi \\
\iint_{(\eta, T) \times \mathbb{R}^{d}} \nabla^{\alpha-1}\left(\left|u_{n}\right|^{m-1} \operatorname{sgn} u_{n}\right) \cdot \nabla \varphi \mathrm{d} t \mathrm{~d} x & \rightarrow \iint_{(\eta, T) \times \mathbb{R}^{d}} \nabla^{\alpha-1}\left(\left|u^{\eta}\right|^{m-1} \operatorname{sgn} u^{\eta}\right) \cdot \nabla \varphi \mathrm{d} t \mathrm{~d} x
\end{aligned}
$$

as $n \rightarrow \infty$ for each $\eta>0$. We can now conclude the proof of Theorem 2.6 through a diagonal procedure.

\section{Appendix A. Proof of Proposition 5.9}

Proof of Proposition 5.9. We fix an arbitrary $p \geq p_{\alpha}=\frac{d}{\alpha-1}$. First, we remark that

$$
\iint\left(\partial_{t} u\right) \phi \mathrm{d} t \mathrm{~d} x+\int|u| \nabla^{\alpha-1} G(u) \cdot \nabla \phi \mathrm{d} t \mathrm{~d} x+\delta \iint \nabla u \cdot \nabla \phi \mathrm{d} t \mathrm{~d} x=0
$$

for all $\phi$ compactly supported in time and in $L^{1}\left((0, T), H^{1, p^{\prime}}\left(\mathbb{R}^{d}\right)\right)$. Recall that we have $\left.\partial_{t} u \in \mathcal{C}[0, T], L^{p}\left(\mathbb{R}^{d}\right)\right)$ for $p \geq p_{\alpha}=\frac{d}{\alpha-1}$ and $\Psi(u)=|u| \nabla^{\alpha-1} G(u) \in L^{\infty}\left((0, T), L^{p}\left(\mathbb{R}^{d}\right)\right)$. To justify the previous equality, it is enough to mollify the function $\phi$ in time and space, and remark that the mollified function $\phi_{\eta}$ satisfies (for $\eta$ small enough) $\phi_{\eta}(0, x)=\phi_{\eta}(T, x)=0, u \phi_{\eta} \in \mathcal{C}^{1}\left((0, T), L^{1}\left(\mathbb{R}^{d}\right)\right)$ and

$$
\iint u\left(\partial_{t} \phi_{\eta}\right) \mathrm{d} t \mathrm{~d} x=-\iint\left(\partial_{t} u\right) \phi_{\eta} \mathrm{d} t \mathrm{~d} x
$$

Letting $\eta \rightarrow 0$ and using the regularity of $\Psi(u)$ yields the desired result.

Next, consider $\phi(\tau, x)=\varphi^{\prime}(u(\tau, x)) \Theta_{\eta}(\tau)$, where $\Theta_{\eta}$ is truncation function in time of $[s, t]$. Remark that $\phi \in L^{1}\left((0, T), H^{1, p^{\prime}}\left(\mathbb{R}^{d}\right)\right)$; indeed, $\varphi^{\prime}(u) \in L^{\infty}\left((0, T), L^{p^{\prime}}\left(\mathbb{R}^{d}\right)\right)$, and we also have for all $w \in\left(H^{1, p} \cap L^{1} \cap L^{\infty}\right)\left(\mathbb{R}^{d}\right)$

$$
\left\|\varphi^{\prime \prime}(w) \nabla w\right\|_{p^{\prime}} \leq\|\nabla w\|_{p}\left\|\varphi^{\prime \prime}(w)\right\|_{q}
$$


with $\frac{1}{q}=1-\frac{2}{p}$. As far as $\Theta_{\eta}$ is concerned, we choose it such that $\Theta_{\eta}(s)=0$ and $\Theta_{\eta}^{\prime}(\tau)=\rho_{\eta}(\tau-s-\eta)-\rho_{\eta}(\tau-t+\eta)$ where $\rho_{\eta}$ is an even mollifier supported in $[-\eta, \eta]$. Now we can write

$$
\begin{aligned}
\iint\left(\partial_{t} u\right) \varphi^{\prime}(u) \Theta_{\eta} \mathrm{d} \tau \mathrm{d} x & =\iint \partial_{t}(\varphi(u)) \Theta_{\eta} \mathrm{d} \tau \mathrm{d} x \\
& =\iint \varphi(u)\left(\rho_{\eta}(\tau-t+\eta)-\rho_{\eta}(\tau-s-\eta)\right) \mathrm{d} \tau \mathrm{d} x
\end{aligned}
$$

Hence,

$$
\iint\left(\partial_{t} u\right) \varphi^{\prime}(u) \Theta_{\eta} \mathrm{d} \tau \mathrm{d} x \rightarrow \int \varphi(u(t, x)) \mathrm{d} x-\int \varphi(u(s, x)) \mathrm{d} x
$$

Moreover,

$$
|u| \nabla \phi(u)=|u| \varphi^{\prime \prime}(u) \nabla u=\nabla(\psi(u)) \quad \text { in } L^{p}\left(\mathbb{R}^{d}\right)
$$

thanks to the Stampacchia theorem $\left(u \in L^{\infty}\left(\mathbb{R}^{d}\right)\right.$ hence $\varphi^{\prime}$ and $\psi$ locally Lipschitz is enough). Hence

$$
\int_{s}^{t} \int|u| \nabla^{\alpha-1} G(u) \cdot \nabla \phi(u) \mathrm{d} \tau \mathrm{d} x=\int_{s}^{t} \int \nabla^{\alpha-1} G(u) \cdot \nabla(\psi(u)) \mathrm{d} x \mathrm{~d} \tau .
$$

It remains to prove that

$$
\int_{s}^{t} \int \psi(u)(-\Delta)^{\frac{\alpha}{2}}(G(u)) \mathrm{d} \tau \mathrm{d} x \leq \int_{s}^{t} \int \nabla^{\alpha-1} G(u) \cdot \nabla(\psi(u)) \mathrm{d} x \mathrm{~d} \tau<\infty .
$$

The last inequality comes from the computations we made above. As far as the second inequality is concerned, we use (1.3) to write

$$
\int_{s}^{t} \int \nabla^{\alpha-1} G(u) \cdot \nabla(\psi(u)) \mathrm{d} x \mathrm{~d} \tau=\lim _{\eta \rightarrow 0} \int_{s}^{t} \iint(G(u)(\tau, x)-G(u)(\tau, y)) F_{\eta}(y-x) \cdot \nabla(\psi(u(\tau, x))) \mathrm{d} x \mathrm{~d} y \mathrm{~d} \tau
$$

where $F_{\eta}$ is defined as follows

$$
F_{\eta}(z)=C_{d, \alpha} \frac{z}{\eta^{d+\alpha}+|z|^{d+\alpha}}
$$

Through an integration by parts, we now get

$$
\begin{aligned}
\int_{s}^{t} \iint(G(u)(\tau, x)-G(u)(\tau, y)) F_{\eta}(y-x) \cdot \nabla(\psi(u(\tau, x))) \mathrm{d} x \mathrm{~d} y \mathrm{~d} \tau & \\
=-\int_{s}^{t} \iint \nabla G(u)(\tau, x) \cdot F_{\eta}(y-x) \psi(u(\tau, x)) \mathrm{d} x \mathrm{~d} y \mathrm{~d} \tau & \\
& +\int_{s}^{t} \iint(u(\tau, x)-u(\tau, y))\left(\nabla \cdot F_{\eta}\right)(y-x) \psi(u(\tau, x)) \mathrm{d} x \mathrm{~d} y \mathrm{~d} \tau .
\end{aligned}
$$

The first term on the right-hand side equals 0 since $F_{\eta}$ is odd. Moreover, we have

$$
\nabla \cdot F_{\eta}(z)=C_{d, \alpha} \frac{\eta^{d+\alpha}+(d+\alpha+1)|z|^{d+\alpha}}{\left(\eta^{d+\alpha}+|z|^{d+\alpha}\right)^{2}} .
$$

Hence, the Fatou lemma yields A.1). The proof of the proposition is now complete.

\section{Appendix B. From t6.6) To The Boundedness of $\kappa_{n}$}

Consider $l_{n}=\log \kappa_{n}$ and write (6.6) as follows

$$
l_{n+1} \leq a_{n}+b_{n} l_{n}
$$


with

$$
\begin{aligned}
& a_{n}=\frac{1}{2^{n+1}\left(\frac{\alpha}{d}+\frac{m-1}{2^{n}}\right)} \log \left(\frac{2^{n}\left(\frac{2 \alpha}{d}+\frac{m-1}{2^{n}}\right)+1}{K_{n}\left(\frac{\alpha}{d}+\frac{m-1}{2^{n}}\right)}\right) ; \\
& b_{n}=1-\frac{m-1}{\frac{\alpha}{d} 2^{n+1}+2(m-1)} .
\end{aligned}
$$

Remark next that

$$
a_{n} \leq C \frac{n}{2^{n}} \quad \text { and } \quad b_{n} \leq 1-\frac{C}{2^{n}} .
$$

In particular

$$
\sum_{n \geq k} a_{n}<\infty \quad \text { and } \quad \prod_{n \geq k} b_{n}<\infty
$$

Using the fact that $b_{n} \leq 1$, we get

$$
l_{n} \leq \sum_{n \geq k} a_{n}+\left(\prod_{n \geq k} b_{n}\right) a_{k_{0}} .
$$

Hence, $l_{n}$ does not blow up, and neither does $\kappa_{n}$.

\section{Appendix C. Proof of Lemma 6.4}

Proof of Lemma 6.4. First, we remark that we can reduce to the case $g(t)=t$ and $K=1$ through a change of variables.

The proof is simple if $f$ is smooth. If $f$ is not, extend $f$ by 0 to $\mathbb{R}$ and consider a mollifier $\rho_{\varepsilon}$. Then write for $t_{1}<t_{2}$,

$$
f\left(t_{1}-s\right)+K \int_{-\infty}^{t_{1}-s} f^{\gamma+1}(\tau) \mathrm{d} \tau \leq f\left(t_{2}-s\right) .
$$

Now integrate against $\rho_{\varepsilon}(s)$ and use the Jensen inequality to get

$$
f_{\varepsilon}\left(t_{1}\right)+K \int_{-\infty}^{t_{1}} f_{\varepsilon}^{\gamma+1}(\tau) \mathrm{d} \tau \leq f_{\varepsilon}\left(t_{2}\right) .
$$

We are now reduced to the case $f=f_{\varepsilon}$, which is smooth. Passing to the limit, the proof is now complete.

\section{REFERENCES}

[1] N. D. Alikakos, An application of the invariance principle to reaction-diffusion equations, J. Differential Equations, 33 (1979), pp. 201-225.

[2] G. Barles, E. Chasseigne, and C. Imbert, On the Dirichlet problem for second-order elliptic integro-differential equations, Indiana Univ. Math. J., 57 (2008), pp. 213-246.

[3] P. Biler, C. Imbert, And G. Karch, Barenblatt profiles for a nonlocal porous medium equation., C. R., Math., Acad. Sci. Paris, 349 (2011), pp. 641-645.

[4] P. Biler, G. Karch, and R. Monneau, Nonlinear diffusion of dislocation density and self-similar solutions, Comm. Math. Phys., 294 (2010), pp. 145-168.

[5] L. Caffarelli and J. L. VÁzquez, Nonlinear porous medium flow with fractional potential pressure, Arch. Ration. Mech. Anal., 202 (2011), pp. 537-565.

[6] L. A. Caffarelli, F. Soria, and J. L. VÁzquez, Regularity of solutions of the fractional porous medium flow. Preprint arXiv:1201.6048, 2012.

[7] L. A. Caffarelli and J. L. VÁzquez, Asymptotic behaviour of a porous medium equation with fractional diffusion, Discrete Contin. Dyn. Syst., 29 (2011), pp. 1393-1404.

[8] J. A. Carrillo, A. Jüngel, P. A. Markowich, G. Toscani, and A. Unterreiter, Entropy dissipation methods for degenerate parabolic problems and generalized Sobolev inequalities, Monatsh. Math., 133 (2001), pp. 1-82.

[9] A. Córdoba And D. CórdobA, A maximum principle applied to quasi-geostrophic equations, Comm. Math. Phys., 249 (2004), pp. 511-528.

[10] A. De Pablo, F. Quirós, A. Rodríguez, And J. L. VÁzquez, A fractional porous medium equation, Adv. Math., 226 (2011), pp. 1378-1409.

[11] —, A general fractional porous medium equation, Comm. Pure Applied Mathematics, 65 (2012), pp. $1242-1284$. 
[12] J. Droniou And C. Imbert, Fractal first order partial differential equations, Arch. Ration. Mech. Anal., 182 (2006), pp. 299331.

[13] B. DYDA, Fractional calculus for power functions and eigenvalues of the fractional laplacian, Fractional Calculus and Applied Analysis, 15 (2012), pp. 535-555.

[14] R. K. Getoor, First passage times for symmetric stable processes in space, Trans. Amer. Math. Soc., 101 (1961), pp. 75-90.

[15] C. Imbert And A. Mellet, Existence of solutions for a higher order non-local equation appearing in crack dynamics, Nonlinearity, 24 (2011), pp. 3487-3514.

[16] G. KARCh, C. MiaO, AND X. XU, On convergence of solutions of fractal Burgers equation toward rarefaction waves, SIAM J. Math. Anal., 39 (2008), pp. 1536-1549.

[17] O. Ladyzhenskaya, V. Solonnikov, and N. Ural'tseva, Linear and quasi-linear equations of parabolic type. Translated from the Russian by S. Smith., Translations of Mathematical Monographs. 23. Providence, RI: American Mathematical Society (AMS). XI, 648 p. , 1968.

[18] V. A. Liskevich And Y. A. Semenov, Some problems on Markov semigroups, in Schrödinger operators, Markov semigroups, wavelet analysis, operator algebras, vol. 11 of Math. Top., Akademie Verlag, Berlin, 1996, pp. 163-217.

[19] W. Magnus, F. Oberhettinger, and R. P. Soni, Formulas and theorems for the special functions of mathematical physics, Third enlarged edition. Die Grundlehren der mathematischen Wissenschaften, Band 52, Springer-Verlag New York, Inc., New York, 1966.

[20] A. PAZY, Semigroups of linear operators and applications to partial differential equations, vol. 44 of Applied Mathematical Sciences, Springer-Verlag, New York, 1983.

[21] J. M. Rakotoson and R. Temam, An optimal compactness theorem and application to elliptic-parabolic systems, Appl. Math. Lett., 14 (2001), pp. 303-306.

[22] T. Runst And W. Sickel, Sobolev spaces of fractional order, Nemytskij operators, and nonlinear partial differential equations, vol. 3 of de Gruyter Series in Nonlinear Analysis and Applications, Walter de Gruyter \& Co., Berlin, 1996.

[23] E. M. Stein, Singular integrals and differentiability properties of functions, Princeton Mathematical Series, No. 30, Princeton University Press, Princeton, N.J., 1970.

[24] E. M. Stein and G. Weiss, Introduction to Fourier analysis on Euclidean spaces, Princeton University Press, Princeton, N.J., 1971. Princeton Mathematical Series, No. 32.

[25] M. E. TAYLOR, Tools for PDE, vol. 81 of Mathematical Surveys and Monographs, American Mathematical Society, Providence, RI, 2000. Pseudodifferential operators, paradifferential operators, and layer potentials.

[26] — Partial differential equations. III: Nonlinear equations. 2nd ed., Applied Mathematical Sciences 117. New York, NY: Springer. xxii, 715 p. , 2011.

[27] J. L. VÁzquez, Smoothing and decay estimates for nonlinear diffusion equations, vol. 33 of Oxford Lecture Series in Mathematics and its Applications, Oxford University Press, Oxford, 2006. Equations of porous medium type.

[28] — The porous medium equation, Oxford Mathematical Monographs, The Clarendon Press Oxford University Press, Oxford, 2007. Mathematical theory.

[29] J. L. VÁzQuEz, Barenblatt solutions and asymptotic behaviour for a nonlinear fractional heat equation of porous medium type. Preprint arXiv:1205.6332v1, 2012.

[30] G. N. Watson, A treatise on the theory of Bessel functions, Cambridge Mathematical Library, Cambridge University Press, Cambridge, 1995. Reprint of the second (1944) edition.

P. Biler: Instytut Matematyczny, Uniwersytet WrocŁawski, Pl. Grunwaldzki 2/4, 50-384 Wroceaw, Poland

E-mail address: Piotr.Biler@math.uni.wroc.pl

C. Imbert: CNRS, UMr 8050, Université Paris-Est Créteil, 61 av. Du Général de Gaulle, 94010 Créteil, Cedex, FRANCE

E-mail address: cyril.imbert@u-pec.fr

$U R L:$ http://www.perso-math.univ-mlv.fr/users/imbert.cyril/

G. Karch: Instytut Matematyczny, Uniwersytet Wroceawski, Pl. Grunwaldzki 2/4, 50-384 WrocŁaW, Poland

E-mail address: Grzegorz.Karch@math.uni.wroc.pl

URL: http://www.math.uni.wroc.pl/ ${ }^{k a r c h}$ 